\title{
A Polysaccharide Derived from a Trichosporon sp. Culture Strongly Primes Plant Resistance to Viruses
}

\author{
Yi-Shu Chiu, ${ }^{1}$ Pi-Yu Chen, ${ }^{1}$ Tung Kuan, ${ }^{2}$ Po-Chuan Wang, ${ }^{1,2}$ Ying-Ju Chen, ${ }^{1,2}$ Yu-Liang Yang, ${ }^{1,+}$ and \\ Hsin-Hung Yeh ${ }^{1,2,+}$ \\ ${ }^{1}$ Agricultural Biotechnology Research Center, Academia Sinica, Taipei 11529, Taiwan; and ${ }^{2}$ Department of Plant Pathology and \\ Microbiology, National Taiwan University, Taipei 10617, Taiwan
}

Accepted 4 June 2018.

\begin{abstract}
Plant viruses cause devastating diseases in plants, yet no effective viricide is available for agricultural application. We screened cultured filtrates derived from various soil microorganisms cultured in vegetable broth that enhanced plant viral resistance. A cultured filtrate, designated $\mathrm{F8}$ culture filtrate, derived from a fungus belonging to the genus Trichosporon, induced strong resistance to various viruses on different plants. Our inoculation assay found the infection rate of Tobacco mosaic virus (TMV)-inoculated Nicotiana benthamiana with F8 culture filtrate pretreatment may decrease to $0 \%$, whereas salicylic acid (SA)-pretreated $N$. benthamiana attenuated TMV-caused symptoms but remained $100 \%$ infected. Tracking Tobacco mosaic virus tagged with green fluorescence protein in plants revealed pretreatment with $\mathrm{F8}$ culture filtrate affected the initial establishment of the virus infection. From F8 culture filtrate, we identified a previously unknown polysaccharide composed of D-mannose, D-galactose, and D-glucose in the ratio 1.0:1.2:10.0 with a $\alpha$-D-1,4-glucan linkage to be responsible for the induction of plant resistance against viruses through priming of SA-governed immune-responsive genes. Notably, F8 culture filtrate only triggered local defense but was much more effective than conventional SA-mediated systematic acquired resistance. Our finding revealed that microbial cultured metabolites provided a rich source for identification of potent elicitors in plant defense.
\end{abstract}

Viruses cause serious diseases in crops, and the lost yield is estimated to total more than $\$ 30$ billion annually (Sastry and Zitter 2014). However, because viruses are intracellular parasites, cost-effectively eliminating viruses from infected plants is difficult. Currently, no curative viricides are available for plant viral disease management. Thus, unlike other pests, if viruses can evade preventive control measures and infect plants, roguing

\section{Yi-Shu Chiu and Pi-Yu Chen contributed equally to this work.}

Nucleotide sequence data is available in GenBank databases under accession number MG757354 and MG752969.

${ }^{\dagger}$ Corresponding authors: Hsin-Hung Yeh; E-mail: hyeh@sinica.edu.tw and Yu-Liang Yang: E-mail: ylyang@gate.sinica.edu.tw

Funding: This work was supported by grants from Academia Sinica.

*The $\boldsymbol{e}$-Xtra logo stands for "electronic extra" and indicates that six supplementary figures, three supplementary tables, and a supplementary text file are published online.

(c) 2018 The American Phytopathological Society is the main option for farmers to prevent further spread of viral disease. Traditional resistance breeding, use of pesticides to control viral vectors, and transgenic approaches to control viral diseases in crops often come at the cost of time, compromised flavor, hazardous concerns to the environment and health, as well as issues associated with genetically modified foods (Herring 2008; Galvez et al. 2014). One way to alleviate this problem is to induce a plant immune response to defend against virus infection.

Plants protect against pest attack by structural barriers or preexisting pest-toxic chemicals (Dangl and Jones 2001). In addition, plants have evolved a complex immune response that can ward off attack by most microbes. Plants can recognize microbe- and pathogen-conserved molecular structures termed microbe- or pathogen-associated molecular patterns (MAMPs/ PAMPs) and induce an immune response (Chisholm et al. 2006; Göhre and Robatzek 2008; Jones and Dangl 2006). An example of MAMPs/PAMPs are bacterial flagellin, chitin, or different glucans that constitute fungal and oomycete cell walls (Boller and Felix 2009; Newman et al. 2013). In addition to MAMPs/ PAMPs, host-derived molecules may be generated during attacks by microbes or pathogens through lytic enzymes or mechanical forces. Some of these molecules may serve as elicitors of a plant defense response (Boller and Felix 2009; Dodds and Rathjen 2010). These molecules are termed danger- or damageassociated molecular patterns (DAMPs) (Boller and Felix 2009; Dodds and Rathjen 2010). MAMPs and DAMPs are perceived by membrane-localized pattern recognition receptors and induce a diverse array of defense responses commonly referred to as pattern-triggered immunity (PTI) (Boller and Felix 2009; Jones and Dangl 2006). PTI can defend against most pathogen infection; however, some plant pathogens have evolved effectors that are injected into the host cells to compromise PTI (Jones and Dangl 2006). To defend against pathogens armed with an effector, plants have evolved resistance proteins to detect these effectors and induce a stronger defense response, termed effector-triggered immunity (ETI) (Jones and Dangl 2006). ETI usually triggers programmed cell death at the site of infection to prevent further spreading of invasive pathogens. Both PTI and ETI are coordinated by phytohormones such as salicylic acid (SA), jasmonic acid (JA), ethylene (ET), and abscisic acid (Glazebrook 2005; Pieterse et al. 2012). The coordination between these hormones activates a specific immune response under PTI and ETI to protect against a particular pathogen (Robert-Seilaniantz et al. 2011; Pieterse et al. 2012). In general, the SA- and JA/ETdependent immune responses are effective against biotrophic and necrotrophic pathogens, respectively (Glazebrook 2005).

In plants, local infection may also induce a systemic plant immune response. Two types of induced systemic immune 
responses, systemic acquired response (SAR) and induced systemic response (ISR), have been reported in plants (Pieterse et al. 2014). ISR is induced by plant growth-promoting rhizobacteria and depends on JA and ET (Conrath 2011; Pieterse et al. 2014). The onset of SAR depends on SA and SAR is efficient against a broad spectrum of pathogens, including viruses (Gozzo and Faoro 2013). SAR is usually induced by pathogens that cause a necrotic lesion resulting from a hypersensitive response during an incompatible interaction or cell death with a compatible interaction (Durrant and Dong 2004; Hammerschmidt 1999).

The systemic immune response is usually associated with a primed state, whereby plants respond either faster, more strongly, or both in activating defense responses when subsequently challenged by pathogens (Conrath et al. 2015; Mauch-Mani et al. 2017). Elevated levels of pattern recognition receptors, dormant signaling enzymes, transcription factors, and alterations in chromatin state have been suggested to provide plants with a memory to establish a defense primed state (Conrath et al. 2015; MauchMani et al. 2017). The immune priming allows plants to better defend against pathogen invasion with lower resistance-associated fitness cost (Conrath et al. 2015; Mauch-Mani et al. 2017; van Hulten et al. 2006; Wang et al. 2015).

Viruses usually trigger the SA-related plant defense response, and exogenous application of SA or its analogs, such as benzothiadiazol-7-carbothioic acid-S-methyl ester (BTH) and acibenzolar$S$-methyl (a derivative of BTH), can trigger the SAR against virus infection (Bektas and Eulgem 2015). However, effectiveness and phytotoxicity hamper the application of SA or its analogs in the field (Alexandersson et al. 2016; Gozzo and Faoro 2013).

In addition to synthetic chemicals, natural PAMPs and DAMPs have been reported to trigger immune responses against virus infection (Wiesel et al. 2014). Of note, each PAMP or DAMP mounts a different plant immune response (Wiesel et al. 2014). Simultaneous application of two typical bacterial PAMPs, flg 22 and lipopolysaccharide, to Arabidopsis thaliana induced a typical SAR response but without necrotic lesions (Mishina and Zeier 2007). Therefore, application of combined PAMPs may provide increased protection with less damage to plants.

In view of the diversity of microorganisms and plants, many PAMPs and DAMPs may still be unidentified. In this study, we screened culture filtrates derived from soil microorganisms (may contain PAMPs) cultured with medium made from weeds and readily obtainable vegetables (may contain DAMPs) to induce effective plant defense against viruses. We obtained a culture filtrate derived from a Trichosporon sp. (F8 culture filtrate) that is much better than SA in inducing plant resistance to different viruses but without a fitness cost. We identified the specific mode of action of the F8 culture filtrate, which induced an unusual priming of SA-governed response genes, as well as the active component of the F8 culture filtrate. Our finding revealed an effective way to induce plant resistance against viruses. Furthermore, our detailed analysis uncovered several unusual features that provide important knowledge for further application of induced resistance against virus infection.

\section{RESULTS}

\section{Screening of culture filtrates derived}

from soil microorganisms for inducing plant resistance against Tobacco mosaic virus (TMV).

To identify PAMPs and DAMPs that can provide plant defense against viruses, we first collected soil samples at different locations and followed a previously reported method for microorganism isolation (Ko et al. 2010). We isolated $199 \mathrm{mi}-$ croorganisms, including 77 actinomycetes, 68 bacteria, and 54 fungi.

We selected TMV and its local lesion host, Nicotiana gluti$n o s a$, as a system for the initial screening. TMV can induce local lesions on $N$. glutinosa. The number and size of lesions

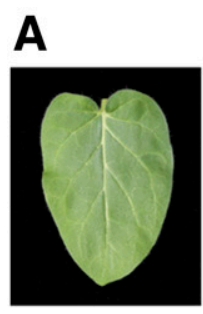

Mock

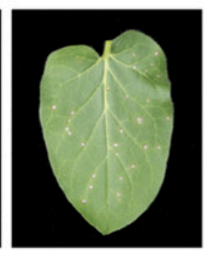

$\mathrm{H}_{2} \mathrm{O}$

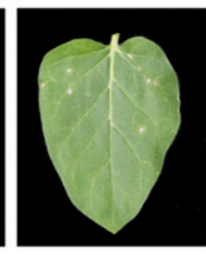

SA

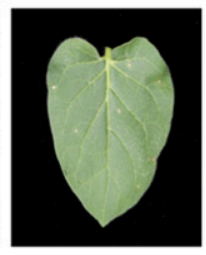

VB

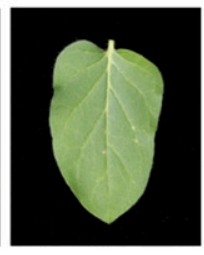

F2

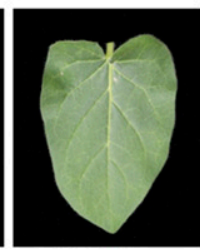

F8

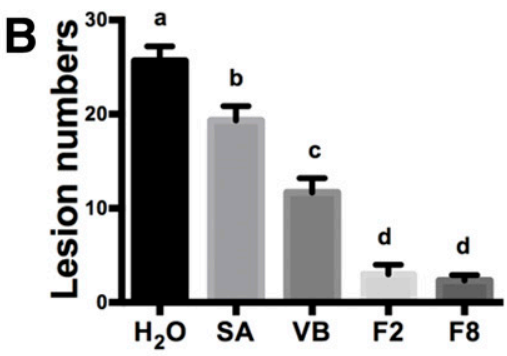

C

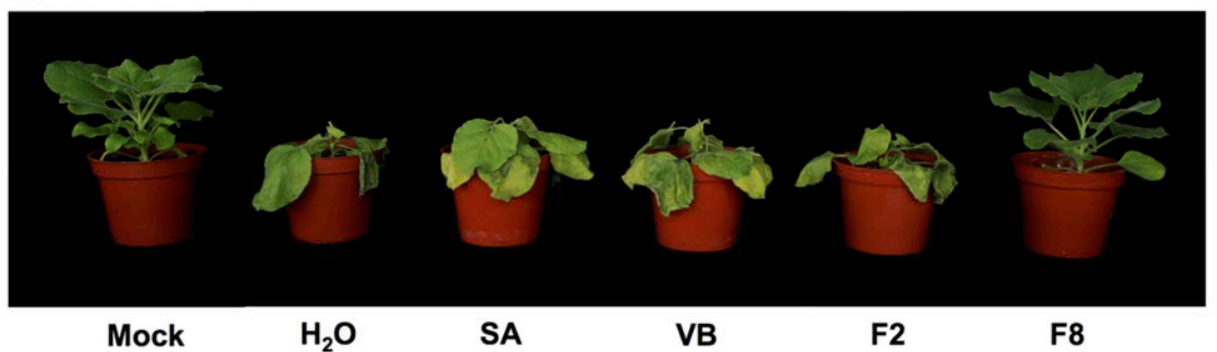

D

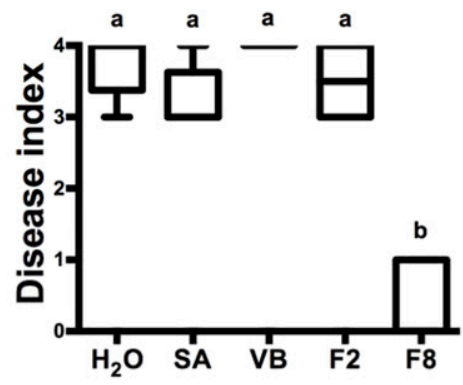

Fig. 1. Lesion number and symptoms induced by Tobacco mosaic virus (TMV) with F2 and F8 culture filtrate pretreatment. A, Nicotiana glutinosa was pretreated with $\mathrm{H}_{2} \mathrm{O}, 5 \mathrm{mM}$ salicylic acid (SA), vegetable broth (VB), or F2 and F8 culture filtrate three times, with a 24-h interval between each spray. Pretreated plants were inoculated with TMV at $24 \mathrm{~h}$ posttreatment (hpt) and untreated plants were inoculated with buffer (mock) for a control. Photos were taken at 5 days postinoculation (dpi). B, Lesion numbers induced by TMV recorded at 5 dpi. Data are means \pm standard deviation from three plants. Different letters above the bars represent significant differences among groups, $P<0.05$ by one-way analysis of variance (ANOVA) with Tukey's posttest. Experiments were repeated three times with similar results. C, $N$. benthamiana was pretreated with $\mathrm{H}_{2} \mathrm{O}, 5 \mathrm{mM} \mathrm{SA}, \mathrm{VB}, \mathrm{F} 2$, or F8 culture filtrate for a total of three times, with a 24-h interval between each spray. All pretreated plants were inoculated with TMV at 24 hpt. Photos of TMV-inoculated plants were taken at 7 dpi. D, Disease index levels induced by TMV were recorded at $7 \mathrm{dpi}$. Box plots represent medians, 25 to 75 percentiles (boxes), and minimum and maximum values (whiskers) from six plants. Different letters above the bars represent significant differences among groups, $P<0.05$ by one-way ANOVA with Tukey's posttest. Experiments were repeated three times with similar results. 
reflects the degree of resistance to TMV (Loebenstein 2009). The soil microorganisms were cultured in vegetable broth (VB) for 14 days, then, culture filtrates were directly sprayed on $N$. glutinosa $(0.4 \mathrm{ml}$ per plant) for a total of three times, with a 24-h interval between each spray. Then, TMV inoculum was rubbed onto leaves of $N$. glutinosa. The lesion number was counted at 5 days postinoculation (dpi). Because VB may contain DAMPs that trigger plant immunity, the results are represented as percent lesion number induced by TMV on plants pretreated with culture filtrates from isolated microorganisms relative to VB-pretreated plants. Plants pretreated with culture filtrates from two fungi (F2 and F8) had fewer lesions than did VB-pretreated plants (Supplementary Fig. S1).

To verify the ability of F2- and F8 culture filtrate to induce resistance in $N$. glutinosa, we treated plants with $\mathrm{H}_{2} \mathrm{O}, \mathrm{SA}, \mathrm{VB}$, or F2 or F8 culture filtrate followed by TMV inoculation. All experiments were repeated at least three times, and the results were consistent, showing much-reduced lesion numbers on $N$. glutinosa pretreated with F2 and F8 culture filtrate (Fig. 1A and B).

\section{F8 culture filtrate induces strong resistance in $N$. benthamiana.}

Besides using N. glutinosa for our initial screening, we also used $N$. benthamiana, a host that is highly susceptible to TMV, for a resistance assay. $N$. benthamiana was pretreated with $\mathrm{H}_{2} \mathrm{O}, \mathrm{SA}$, $\mathrm{VB}$, or $\mathrm{F} 2$ or F8 culture filtrate as for pretreatment of $N$. glutinosa. All treated plants were inoculated with TMV inoculum made from lyophilized leaves of TMV-infected $N$. benthamiana. Plants pretreated with $\mathrm{H}_{2} \mathrm{O}, \mathrm{SA}, \mathrm{VB}$, and $\mathrm{F} 2$ culture filtrate showed severe symptoms; however, most plants with F8 culture filtrate pretreatment remained healthy-looking (Fig. 1C and D). With reverse transcription-polymerase chain reaction (RT-PCR) to detect TMV on all treated plants, the infection rate was much reduced with F8 culture filtrate as compared with $\mathrm{H}_{2} \mathrm{O}, \mathrm{SA}, \mathrm{VB}$, or F2 culture filtrate pretreatment (Table 1). In our repeat experiments, the infection rate of TMV-inoculated plants with F8 culture filtrate pretreatment even decreased to $0 \%$ (Table 1). In addition, plants with F8 culture filtrate pretreatment and TMV infection showed no obvious symptoms (Fig. 1D). We prepared at least seven different batches of F8 culture filtrate, and the ability to induce resistance against TMV was consistent between different batches (Table 1). Of note, F2 culture filtrate did not induce resistance to TMV in N. benthamiana (Table 1; Fig. $1 \mathrm{C}$ and D), so our later experiments focused on F8 culture filtrate.

In addition to using inoculum made from lyophilized leaves of TMV-infected $N$. benthamiana, we also used different concentrations $(1,10$, and $50 \mu \mathrm{g} / \mathrm{ml})$ of purified TMV particles as inoculum to inoculate $N$. glutinosa and $N$. benthamiana pretreated with $\mathrm{H}_{2} \mathrm{O}, \mathrm{SA}, \mathrm{VB}$, or F8 culture filtrate (Figs. 2 and $3)$. The results are consistent with previous results, which showed that TMV particle-inoculated N. glutinosa and N. benthamiana showed fewer lesions and less disease severity in F8 culture filtrate-treated N. glutinosa and N. benthamiana, respectively (Figs. 2 and 3). The results also showed that increased TMV particle concentration positively correlated with increased lesion number and disease severity on leaves of F8 culture filtrate-treated $N$. glutinosa, and $N$. benthamiana, respectively. The infection rate of TMV on $N$. benthamiana was also detected by use of quantitative (q)RT-PCR (Table 2). Reduced infection rate was only observed on F8 culture filtrate-treated $N$. benthamiana when TMV particles at the concentration of $1 \mu \mathrm{g} / \mathrm{ml}$ was used as inoculum (Table 2). Collectively, the data indicated that F8 culture filtrate can induce plant resistance to TMV infection, and an increased concentration of TMV inoculum will increase the infectivity.

\section{More resistance with $\mathbf{F 8}$ culture filtrate than $\mathrm{F} 8$ culture pellets.}

To investigate whether pellets of F8 culture also induce plant resistance against TMV, pellets of F8 culture were resuspended with $\mathrm{H}_{2} \mathrm{O}$ (same volume as the original $\mathrm{F} 8$ culture). The resuspended pellet was squeezed twice in a high-pressure homogenizer to break down the fungi cells. The final F8 culture pellet suspension as well as $\mathrm{H}_{2} \mathrm{O}, \mathrm{SA}, \mathrm{VB}$, or F8 culture filtrate were used to pretreat $N$. benthamiana, followed by inoculation with TMV as previously described. F8-pellet-treated plants showed mild symptoms, with no obvious symptoms seen with F8 culture filtrate pretreatment (Supplementary Fig. S2A and B). Even when the concentration of F8 pellets was doubled, the TMV-induced symptoms were still more severe than with F8 culture filtrate pretreatment.

\section{Treating local leaves with $\mathrm{F8}$ culture filtrate does not protect against inoculation of the upper leaves.}

To investigate whether F8 culture filtrate can protect plants systemically, we treated only the lower leaves (local) of $N$. benthamiana (method described above) with $\mathrm{H}_{2} \mathrm{O}, \mathrm{SA}, \mathrm{VB}$, or F8 culture filtrate and inoculated TMV in the upper untreated (systemic) leaves. As a control, we used F8 culture filtrate to treat whole plants followed by TMV inoculation. Whole plants pretreated with F8 culture filtrate showed much resistance to TMV (Fig. 4A and B); however, all plants with pretreatment of only local leaves showed severe viral symptoms (Fig. 4A and B). Therefore, F8 culture filtrate provides strong resistance in only treated leaves.

In another set of experiments, we pretreated local leaves of plants with $\mathrm{H}_{2} \mathrm{O}, \mathrm{SA}, \mathrm{VB}$, or $\mathrm{F} 8$ culture filtrate but did not inoculate them with TMV. We collected both the local and upper untreated (systemic) leaves at $24 \mathrm{~h}$ posttreatment (hpt) to analyze the expression of the $P R-1$ gene (a SAR marker gene) (Zheng et al. 2015). PR-1 gene expression was detected in SAand VB- but not F8 culture filtrate-treated local leaves (Fig.

Table 1. The infection rate of Tobacco mosaic virus (TMV) on Nicotiana benthamiana with different pretreatments

\begin{tabular}{|c|c|c|c|c|c|c|c|c|c|c|}
\hline \multirow[b]{2}{*}{ Pretreatment $^{a}$} & \multirow[b]{2}{*}{ Inoculum $^{b}$} & \multicolumn{8}{|c|}{ Experiment $^{c}$} & \multirow[b]{2}{*}{ Total } \\
\hline & & 1 & 2 & 3 & 4 & 5 & 6 & 7 & 8 & \\
\hline - & Buffer & $0 / 5$ & $0 / 5$ & $0 / 5$ & $0 / 5$ & $0 / 5$ & $0 / 5$ & $0 / 5$ & $0 / 5$ & $0 / 40$ \\
\hline $\mathrm{H}_{2} \mathrm{O}$ & TMV & $5 / 5$ & $5 / 5$ & $5 / 5$ & $5 / 5$ & $5 / 5$ & $5 / 5$ & $5 / 5$ & $5 / 5$ & $40 / 40$ \\
\hline $\mathrm{SA}$ & TMV & $5 / 5$ & $5 / 5$ & $4 / 5$ & $5 / 5$ & $5 / 5$ & $5 / 5$ & $5 / 5$ & $5 / 5$ & $39 / 40$ \\
\hline VB & TMV & $5 / 5$ & $5 / 5$ & $5 / 5$ & $4 / 5$ & $5 / 5$ & $5 / 5$ & $5 / 5$ & $5 / 5$ & $39 / 40$ \\
\hline $\mathrm{F} 2$ & TMV & $5 / 5$ & $5 / 5$ & $5 / 5$ & - & - & - & - & - & $15 / 15$ \\
\hline F8 & TMV & $1 / 5$ & $0 / 5$ & $0 / 5$ & $1 / 5$ & $0 / 5$ & $1 / 5$ & $0 / 5$ & $0 / 5$ & $3 / 40$ \\
\hline
\end{tabular}

a Plants pretreated with $\mathrm{H}_{2} \mathrm{O}, 5 \mathrm{mM}$ salicylic acid (SA), vegetable broth (VB), and culture filtrate derived from F2 or F8 culture filtrate (F2 or F8).

b Inoculums were made from lyophilized TMV-inoculated leaves.

${ }^{c}$ Number of plants detected with TMV to total TMV-inoculated plants. All leaf samples were collected at 7 days postinoculation, and TMV was detected by reverse transcription-polymerase chain reaction. Experiments were repeated at least eight times. The F8 culture filtrate used in experiments 1 and 2 is from the same batch of preparation; F8 culture filtrate used in experiments 3 to 8 was prepared individually. 
4C). However, only SA-treated plants (positive control) showed increased $P R-1$ gene expression on systemic leaves (Fig. 4D). In addition, we collected leaves from plants with whole plants pretreated with $\mathrm{H}_{2} \mathrm{O}, \mathrm{SA}, \mathrm{VB}$, or F8 culture filtrate. Consistently,
$P R-1$ gene induction was detected in leaves of whole plants pretreated with SA and VB but not whole plants pretreated with F8 culture filtrate at $24 \mathrm{hpt}$ (Fig. 4E). Thus, F8 culture filtrate does not induce canonical SAR.

\section{A Mock}
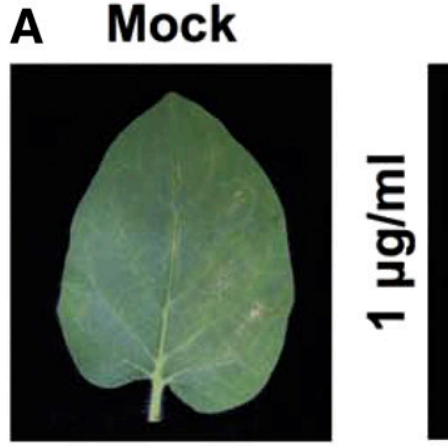

SA VB

F8
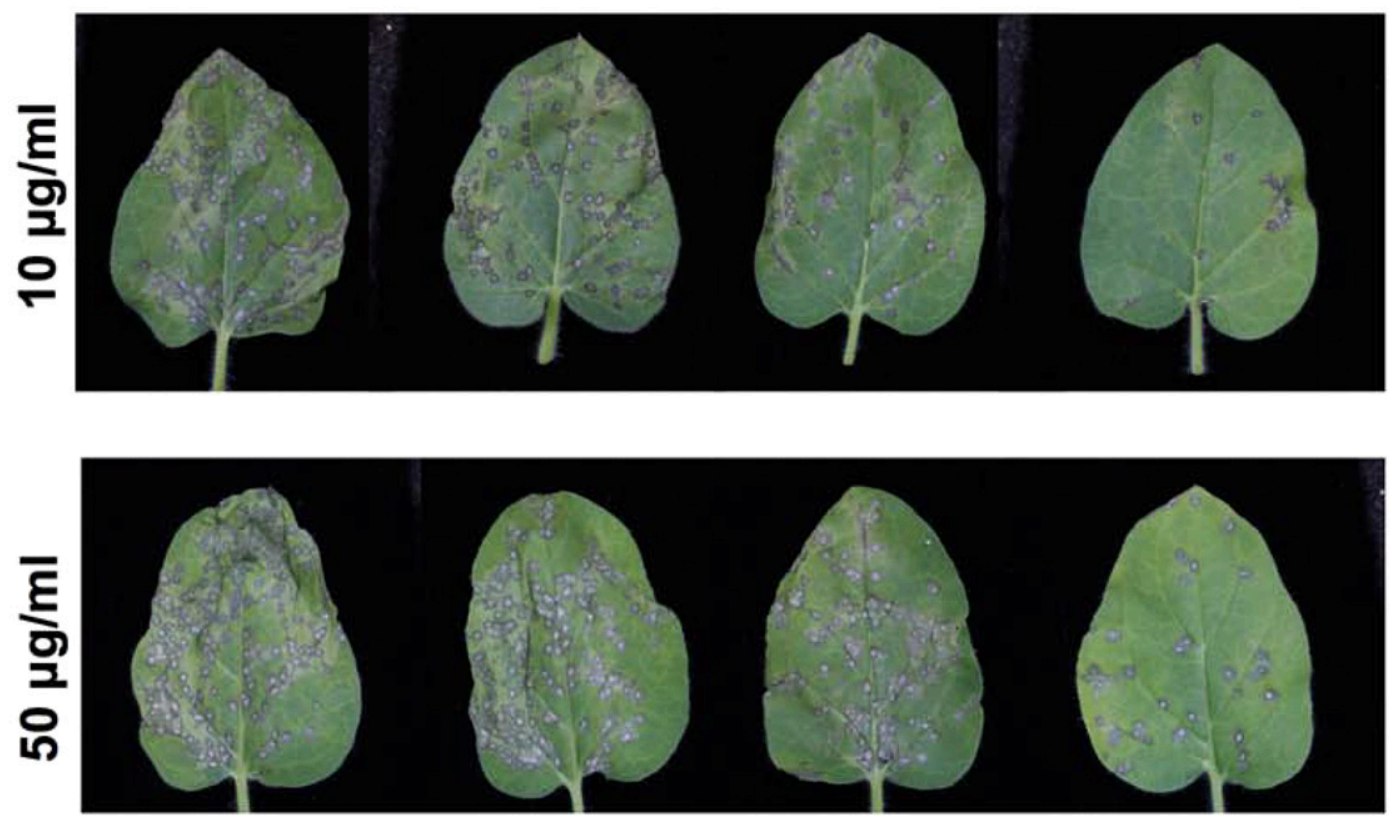

B

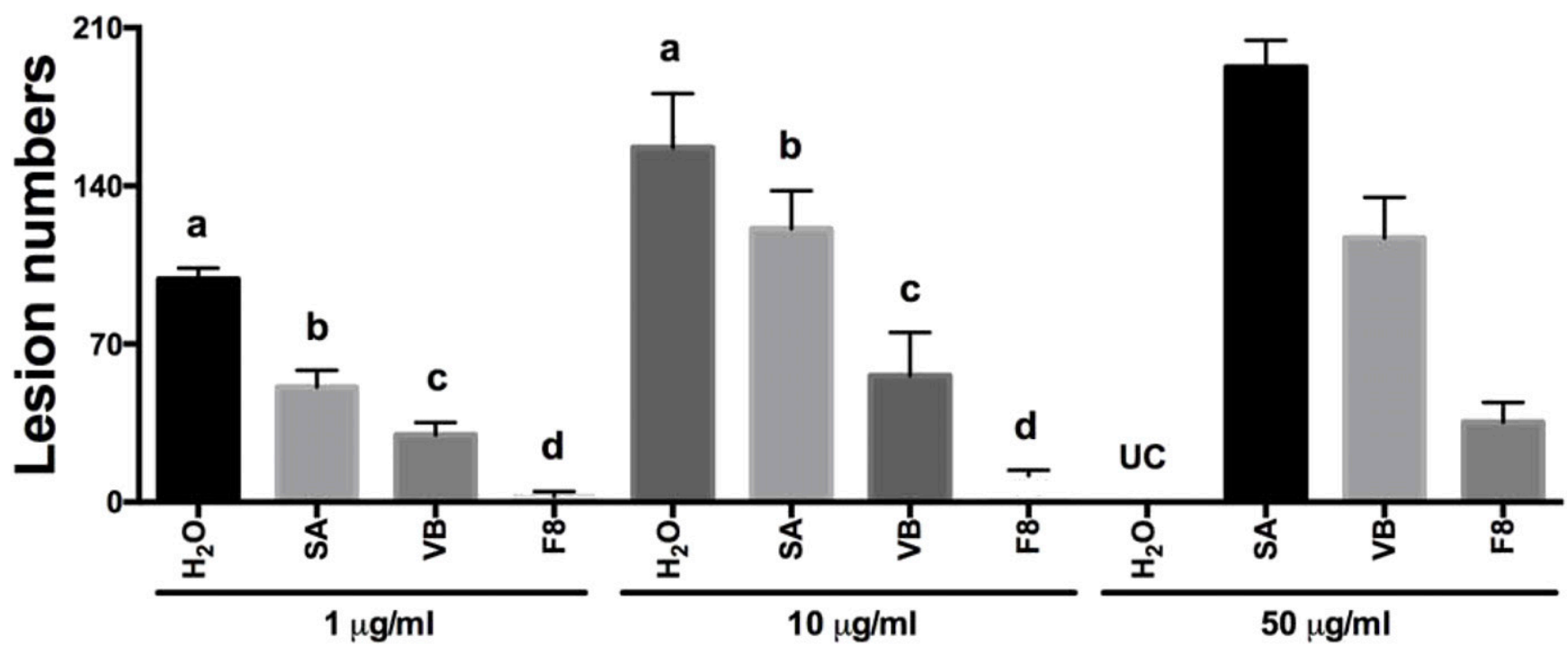

Fig. 2. Lesion number on F8 culture filtrate-treated Nicotiana glutinosa inoculated with purified Tobacco mosaic virus (TMV) particles. A, N. glutinosa was pretreated with $\mathrm{H}_{2} \mathrm{O}, 5 \mathrm{mM}$ salicylic acid (SA), vegetable broth (VB), and F8 culture filtrate three times, with a 24-h interval between each spray. Pretreated plants were inoculated with $1-\mu \mathrm{g} / \mathrm{ml}, 10-\mu \mathrm{g} / \mathrm{ml}$, or $50-\mu \mathrm{g} / \mathrm{ml}$ concentrations of TMV particles at $24 \mathrm{~h}$ posttreatment and untreated plants were inoculated with buffer (mock) for a control. Photos were taken at 5 days postinoculation (dpi). B, Lesion numbers induced by TMV particles was recorded at 5 dpi. UC $=$ uncountable. Data are means \pm standard deviation from three plants. Different letters above the bars represent significant differences among groups, $P<0.05$ by one-way analysis of variance, with Tukey's posttest. 
To analyze whether F8 culture induces stronger resistance on TMV-inoculated leaves to infection, we inoculated TMV $(1 \mu \mathrm{g} / \mathrm{ml})$ on $\mathrm{H}_{2} \mathrm{O}$ - or $\mathrm{F} 8$ culture filtrate-pretreated leaves of $N$. benthamiana and collected TMV-inoculated leaves at 24 , 48,72 , and $96 \mathrm{~h}$ postinoculation (hpi) to analyze the TMV accumulation and expression of the $P R-1$ gene by qRT-PCR. The data showed that, in comparison with $\mathrm{H}_{2} \mathrm{O}$ treated plants, decreased TMV accumulation and increased PR1 expression were observed on F8 culture filtrate-treated plants (Fig. 4F).

A

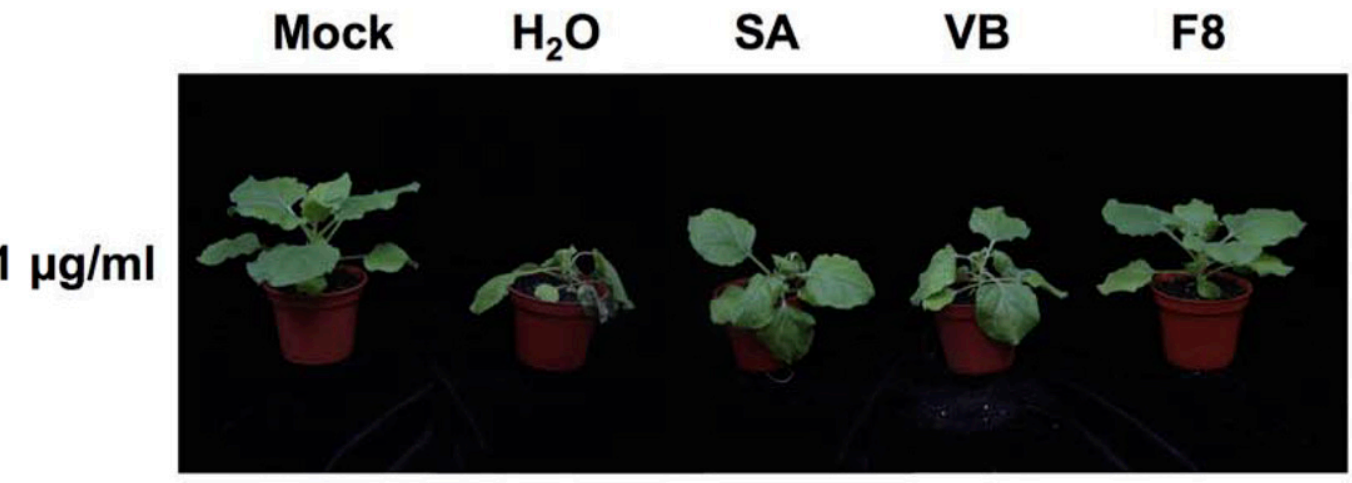

$10 \mu \mathrm{g} / \mathrm{ml}$

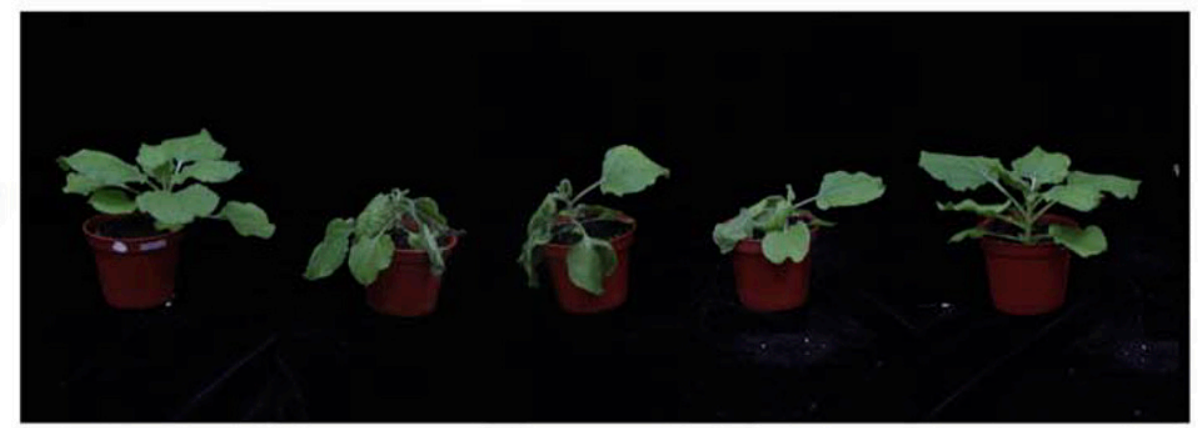

$50 \mu \mathrm{g} / \mathrm{ml}$

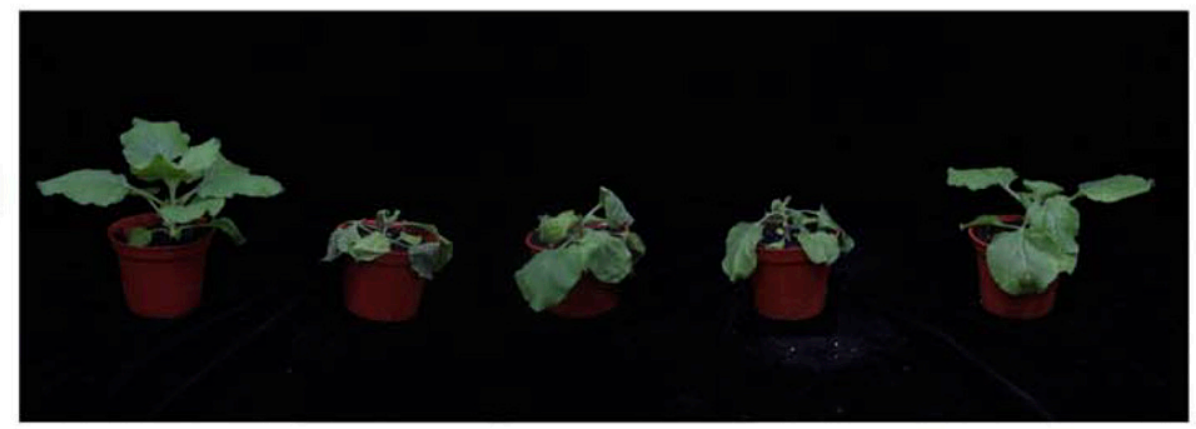

B

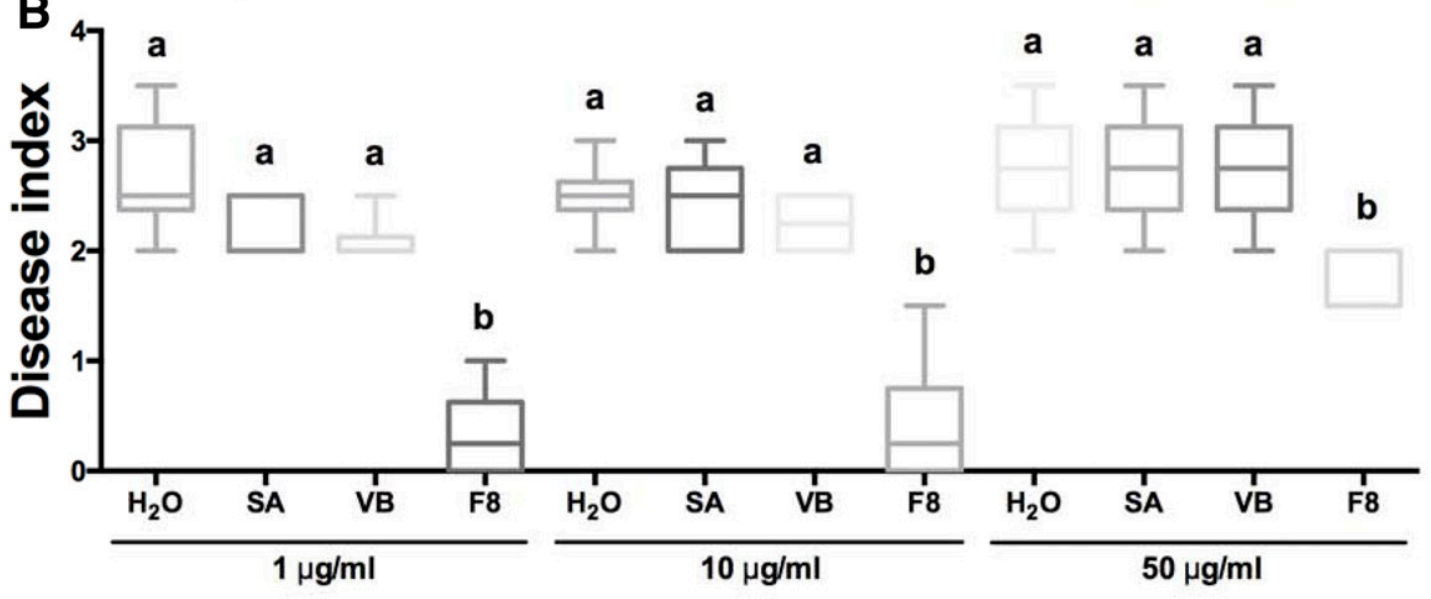

Fig. 3. Symptoms on F8 culture filtrate-treated Nicotiana benthamiana inoculated with purified Tobacco mosaic virus (TMV) particles. A, N. benthamiana was pretreated with $\mathrm{H}_{2} \mathrm{O}, 5 \mathrm{mM}$ salicylic acid (SA), vegetable broth (VB), or F8 culture filtrate (F8) three times at 24-h intervals. All plants were inoculated with $1-\mu \mathrm{g} / \mathrm{ml}, 10-\mu \mathrm{g} / \mathrm{ml}$, or $50-\mu \mathrm{g} / \mathrm{ml}$ concentrations of TMV particles at $24 \mathrm{~h}$ posttreatment. Photos were taken at 5 days postinoculation (dpi). B, Disease index levels induced by TMV were recorded at 5 dpi. Box plots represent medians, 25 to 75 percentiles (boxes), and minimum and maximum values (whiskers) from six plants. Different letters above the bars represent significant differences among groups, $P<0.05$ by one-way analysis of variance, with Tukey's posttest. 
Treatment with F8 culture filtrate affects the establishment of initial TMV infection.

To analyze which virus infection steps are affected in F8 culture filtrate-treated plants, we used TMV tagged with green fluorescence protein (GFP) to inoculate $N$. benthamiana and monitor the infection of TMV. Similar to our previous result, the infection rate of TMV-GFP was much reduced in $N$. benthamiana pretreated with F8 culture filtrate as compared with

Table 2. The infection rate of Tobacco mosaic virus (TMV) particles on Nicotiana benthamiana with different pretreatments ${ }^{\text {a }}$

\begin{tabular}{|c|c|c|c|c|c|c|}
\hline \multirow[b]{2}{*}{ Pretreatment $^{b}$} & \multirow[b]{2}{*}{ Inoculum } & \multicolumn{3}{|c|}{ Experiment 1} & \multirow{2}{*}{$\frac{\text { Exp. } 2}{1 \mu \mathrm{g} / \mathrm{ml}}$} & \multirow{2}{*}{$\frac{\text { Exp. } 3}{1 \mu g / m l}$} \\
\hline & & $1 \mu \mathrm{g} / \mathrm{ml}$ & $10 \mu \mathrm{g} / \mathrm{ml}$ & $50 \mu \mathrm{g} / \mathrm{ml}$ & & \\
\hline- & Buffer & & $0 / 5$ & & $0 / 3$ & $0 / 3$ \\
\hline $\mathrm{H}_{2} \mathrm{O}$ & TMV particle & $6 / 6$ & $6 / 6$ & $6 / 6$ & $6 / 6$ & $9 / 9$ \\
\hline SA & TMV particle & $6 / 6$ & $6 / 6$ & $6 / 6$ & $6 / 6$ & $15 / 15$ \\
\hline VB & TMV particle & $6 / 6$ & $6 / 6$ & $6 / 6$ & $6 / 6$ & $15 / 15$ \\
\hline F8 & TMV particle & $4 / 6$ & $6 / 6$ & $6 / 6$ & $3 / 6$ & $6 / 15$ \\
\hline
\end{tabular}

a Plants were inoculated with difference concentrations of TMV particles. Results are shown as number of plants detected with TMV to total TMV inoculated plants. All leaf samples were collected at 7 days postinoculation, and TMV was detected by reverse transcription-polymerase chain reaction.

b Plants pretreated with $\mathrm{H}_{2} \mathrm{O}, 5 \mathrm{mM}$ salicylic acid (SA), vegetable broth (VB), and F8 culture filtrate (F8).

A

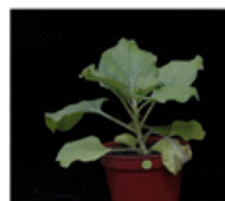

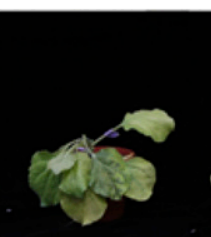

$\mathrm{H}_{2} \mathrm{O}^{\mathrm{L}}$

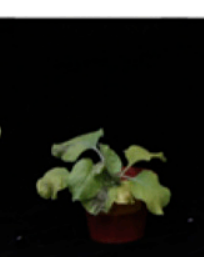

SA $^{\mathrm{L}}$

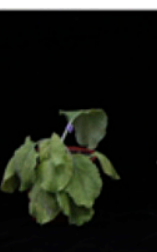

VBL
F8
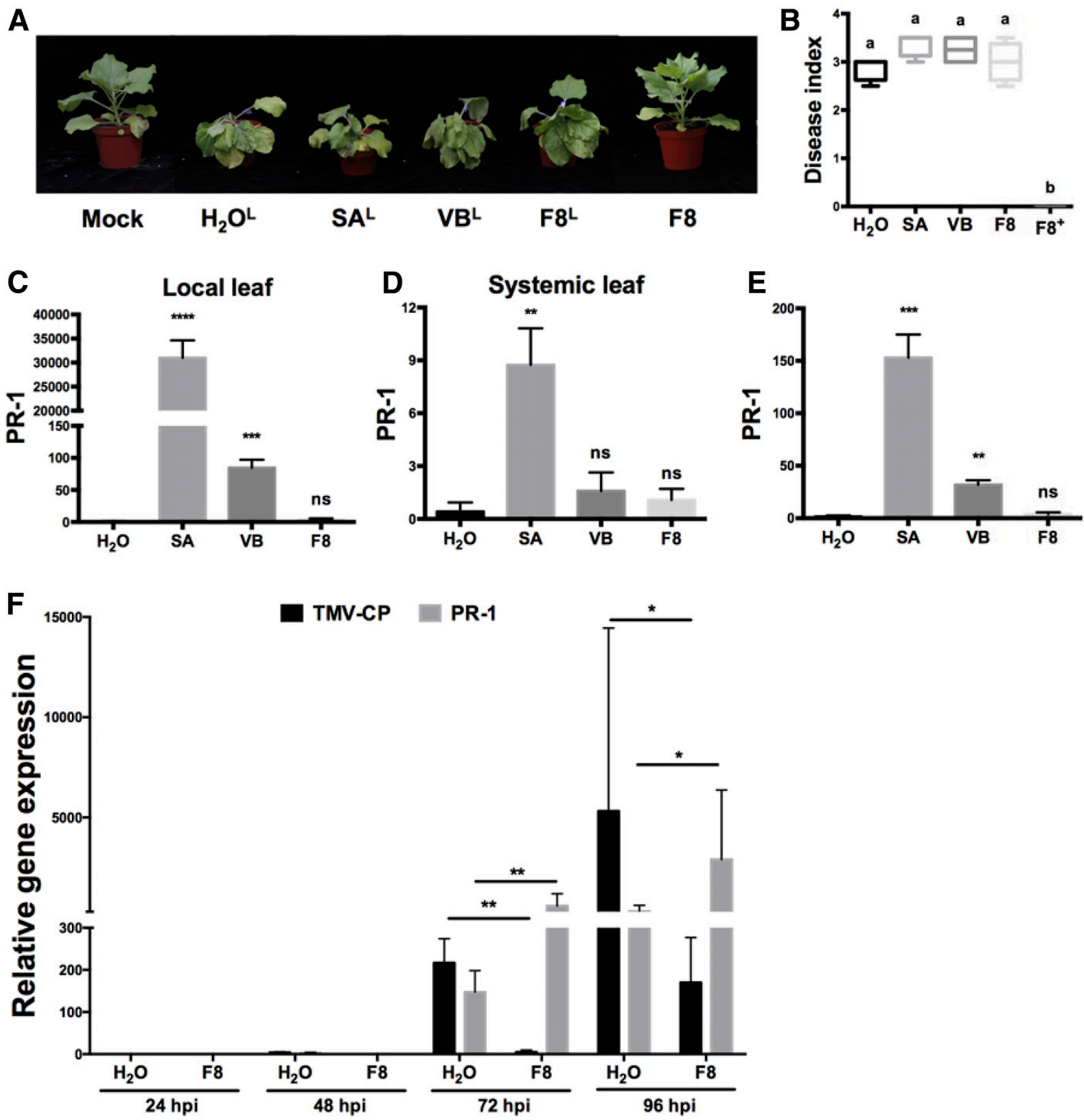
$\mathrm{H}_{2} \mathrm{O}$, SA, or VB (Supplementary Table S2). In addition, with TMV-GFP infection, the number of initial infection foci was reduced with both SA and F8 culture filtrate pretreatment (Fig. 5A and B). To compare TMV-GFP accumulation in each foci, we used a puncher to collect GFP foci under UV light and used qRT-PCR to quantify the TMV-GFP expression. The mean TMV accumulation per foci was similar with $\mathrm{H}_{2} \mathrm{O}, \mathrm{SA}, \mathrm{VB}$, and F8 culture filtrate pretreatment (Fig. 5C).

To understand whether the cell-to-cell movement of TMV was affected with F8 culture filtrate pretreatment, we measured the average size of TMV-GFP infection foci at 5 and $7 \mathrm{dpi}$. In TMV-GFP-infected plants, the mean size of infection foci did not differ between plants with different pretreatment at 5 and 7 dpi $(P<0.05)$ (Fig. 5A and D).

To analyze whether long-distance movement is affected by F8 culture filtrate pretreatment, we measured the average time for TMV-GFP to move to apical leaves. The mean time (days) for TMV to move to apical leaves was similar with different pretreatments (Fig. 5E and F).

\section{F8 culture filtrate induces resistance \\ to Turnip mosaic virus (TuMV) in Brassica juncea.}

To analyze whether F8 culture filtrate induced resistance to different viruses in other plants, we treated plants from an economically important vegetable, Brassica juncea, with $\mathrm{H}_{2} \mathrm{O}, \mathrm{VB}$, and F8 culture filtrate, using the method mentioned above, and inoculated the plants with TuMV. TuMV induced severe mosaic and stunting symptoms in plants without any treatment or with VB pretreatment or induced severe mosaic symptoms in plants with $\mathrm{H}_{2} \mathrm{O}$ pretreatment (Fig. 6A); however, F8 culture filtrate pretreatment did not confer obvious symptoms (Fig. 6A and B).

\section{F8 culture filtrate pretreatment does not cause resistance-associated fitness cost.}

To analyze whether F8 culture filtrate pretreatment reduces resistance-associated fitness cost in plants, we analyzed the fresh weight and leaf area in untreated and F8 culture filtratetreated N. benthamiana and Brassica juncea. Both F8 culture filtrate-treated and untreated $N$. benthamiana and Brassica juncea showed similar leaf area and fresh weight (Fig. 6C and D).

\section{F8 fungi is most related to Trichosporon scarabaeorum.}

To identify the F8 fungi, we designed primers (Supplementary Table S1) to amplify the D1/D2 domain and the intergenic transcribed spacer (ITS) regions of the 26S rDNA (Scorzetti et al. 2002). Phylogenetic analysis was performed by neighborjoining (NJ) and maximum likelihood (ML) methods. The results of both methods were similar and indicated that $\mathrm{F} 8$ fungi is related to T. scarabaeorum (Fig. 7A and B).

F8 is a yeast-like fungus. After culturing the fungi on PDA plate for 15 days, the colony diameter grew to 54 to $57 \mathrm{~mm}$. The F8 colony is beige butter-like and wrinkled on PDA but silk-like and flat on V-8 agar plate (Fig. 7C). Under microscope observation, F8 generated arthroconidia. The arthroconidia were hyaline, short rod-shaped, and obovate (Fig. 7C). Together, molecular phylogenetic analysis of the D1/D2 domain, ITS, and morphological features showed that our identified F8 fungus belongs to the basidiomycetous yeast T. scarabaeorum.

\section{A fraction of polysaccharide derived from $F 8$ culture filtrate is the major active component for inducing plant virus resistance.}

To identify one or more active functional compounds in F8 culture filtrate that are responsible for inducing plant resistance, we separated the F8 culture filtrate by size-exclusion chromatography. Eight fractions (F8-fr1 to F8-fr8) were eluted by $\mathrm{H}_{2} \mathrm{O}$ with a Sephadex LH20 column. Each fraction was analyzed by matrix-assisted laser desorption-ionization time of flight mass spectrometry (MALDI-TOF MS), and the activity in inducing resistance against TMV was initially analyzed in $N$. glutinosa. Our repeat experiments indicated that the second fraction (F8-fr2) induced the strongest antiviral activity in $N$. glutinosa. To determine whether F8-fr2 also induced strong resistance in $N$. benthamiana, we treated $N$. benthamiana with F8-fr1 to F8-fr4, then, inoculated plants with TMV (Supplementary Fig. S3). Consistently, F8-fr2 induced the strongest antiviral activity in $N$. benthamiana (Supplementary Table S3), although the F8 culture filtrate still induced the best resistance.

On MALDI-TOF MS, F8-fr2 showed a polysaccharide signal, with no obvious signals observed after F8-fr4. To purify F8-fr2 polysaccharides, we created three consecutive precipitations with increasing concentrations of ethanol $(20,50$, and $80 \%$ ). The $\mathrm{H}_{2} \mathrm{O}$-resuspended pellets derived from $80 \%$ ethanol precipitation (F8-fr2-80\% EtOH ppt) exhibited the strongest antiviral activity (Fig. 8A and B). Results of MALDI-TOF MS analysis suggested that F8-fr2-80\% EtOH ppt mainly

Fig. 4. F8 culture filtrate does not confer systemic protection against Tobacco mosaic virus (TMV) or conical systemic acquired resistance (SAR) in Nicotiana benthamiana. The lower three leaves (local) of $N$. benthamiana were pretreated with $\mathrm{H}_{2} \mathrm{O}\left(\mathrm{H}_{2} \mathrm{O}^{\mathrm{L}}\right), 5 \mathrm{mM}$ salicylic acid $\left(\mathrm{SA}^{\mathrm{L}}\right)$, vegetable broth $\left(\mathrm{VB}{ }^{\mathrm{L}}\right)$, or $\mathrm{F} 8$ culture filtrate $\left(\mathrm{F}^{\mathrm{L}}\right)$ three times, with a 24 -h interval between each spray. The three upper leaves (systemic) were inoculated with TMV at 2 days posttreatment. Whole plants treated with F8 culture filtrate (F8) three times, with a 24-h interval between each spray, was used as control. A, Photo taken at 7 days postinoculation and $\mathbf{B}$, the level of disease induced by TMV was recorded. Box plots represent medians, 25 to 75 percentiles (boxes), and minimum and maximum values (whiskers) from five plants. Different letters above the bars represent significant differences among groups, $P<0.05$ by one-way analysis of variance, with Tukey's posttest. Experiments were repeated at least three times with similar results. For expression analysis of SAR marker gene $P R-1$, local leaves of plants were pretreated with $\mathrm{H}_{2} \mathrm{O}, 5 \mathrm{mM} \mathrm{SA}, \mathrm{VB}$, or F8 culture filtrate three times, with a 24-h interval between each spray. After treatment, C, local leaves and D, systemic leaves were collected at 24 h posttreatment (hpt). Whole plants treated with $\mathrm{H}_{2} \mathrm{O}, 5 \mathrm{mM} \mathrm{SA}$, VB, or F8 culture filtrate were controls. The relative expression of $P R-1$ was detected by quantitative reverse transcription-polymerase chain reaction (qRT-PCR). Data are means \pm standard deviation (SD) from three plants. Student's $t$ test was used to analyze the data between indicated treatment and the $\mathrm{H}_{2} \mathrm{O}$-pretreated control group. Asterisks indicate significant differences between $\mathrm{H}_{2} \mathrm{O}$ - or F8-treated plants (one asterisk [*] indicates $P<0.05$, two [**] $P<0.01$ ). ns $=$ not significant. Experiments were repeated at least three times with similar results. E, The whole plant was pretreated with $\mathrm{H}_{2} \mathrm{O}, 5 \mathrm{mM} \mathrm{SA}$, VB, or F8 three times, with a 24 -h interval between each spray. Leaves were collected at $24 \mathrm{hpt}$. The relative expression of $P R-1$ was detected by qRT-PCR. Data are means \pm SD from three plants. Student's $t$ test was used to analyze data between the indicated treatment and the $\mathrm{H}_{2} \mathrm{O}$-pretreated control group. Asterisks indicate significant differences between $\mathrm{H}_{2} \mathrm{O}$ - or F8-treated plants (* indicates $P<0.05, * * P<0.01$ ). ns $=$ not significant. Experiments were repeated at least three times with similar results. The whole plant was pretreated with $\mathrm{H}_{2} \mathrm{O}$ or F8 three times, with a 24-h interval between each spray. Pretreated plants were inoculated with TMV at 24 hpt. Inoculated leaves were collected at 24 , 48,72 , and $96 \mathrm{~h}$ postinoculation (hpi). The relative expression of $P R-1$ was detected by qRT-PCR. Data are means \pm SD from three to five plants. Asterisks indicate significant differences between $\mathrm{H}_{2} \mathrm{O}$ - or F8-treated plants ( $*$ indicates $P<0.05$, $* * P<0.01$ ). ns $=$ not significant. F, The whole plant was pretreated with $\mathrm{H}_{2} \mathrm{O}$ or $\mathrm{F} 8$ three times, with a 24-h interval between each spray. Pretreated plants were inoculated with TMV at 24 hpt. Inoculated leaves were collected at $24,48,72$, and 96 hpi. The relative expression of $P R-1$ was detected by qRT-PCR. Data are means \pm SD from three to five plants. Student's $t$ test was used to analyze the data between $\mathrm{F} 8$ treatment and $\mathrm{H}_{2} \mathrm{O}$-pretreated control group. Asterisks indicate significant differences between $\mathrm{H}_{2} \mathrm{O}$ - or F8-treated plants (* indicates $P<0.05$, ** $P<0.01$ ). ns $=$ not significant. 
consists of polysaccharides (Fig. 8C). F8-fr2-80\% EtOH ppt was further separated by high-performance liquid chromatography (HPLC) into three peaks (*1 to *3) (Fig. 8D). The *2 peak (designated F8-polysaccharide) showed the strongest antiviral activity (Fig. 8A and B). The sugar composition of F8-polysaccharide was analyzed by gas chromatography (GC)-MS, which revealed D-mannose, D-galactose, and Dglucose in the ratio 1.0:1.2:10.0. Linkage analysis with GC-MS and nuclear magnetic resonance (NMR) analysis suggested the F8-polysaccharide is mainly $\alpha$-D-1,4-glucan (Fig. 8E and F). HPLC analysis revealed the molecular weight of the F8-polysaccharide as 4.2 to $12.6 \mathrm{kDa}$ (Supplementary Fig. S4).

\section{F8-polysaccharide induces priming}

of SA-governed immune response genes.

To analyze how F8-polysaccharide induces plant viral resistance, we measured the expression of SA-, JA-, and ET-responsive immune marker genes in $\mathrm{H}_{2} \mathrm{O}$ - and $\mathrm{F} 8$-polysaccharide-treated plants (Cortes-Barco et al. 2010; Milling et al. 2011; Zhu et al. 2014). We first confirmed the induction of marker genes induced by
SA ( $P R-1$ and $P R-2)$, JA ( $P D F-1.2$ and $P R-4)$, and ET (Osm and $E R E B P$ ) in $N$. benthamiana (Supplementary Fig. S5A). Plants were pretreated with $\mathrm{H}_{2} \mathrm{O}$ and F8-polysacccharide three times, with a 24-h interval between each spray, as described previously, then, leaves were collected from one set of individual plants at $0,12,24,36,48,72$, and 96 hpt. In another set of plants with $\mathrm{H}_{2} \mathrm{O}$ and F8-polysacccharide pretreatment, TMV was inoculated at $24 \mathrm{hpt}$ and the inoculated leaves from individual plants were collected at $0,12,24,48$, and $72 \mathrm{hpi}$. We detected the accumulation of TMV and the expression of SA-, JA-, and ET-responsive immune marker genes (Fig. 9A to D). Consistently, TMV accumulation at 48 and 72 hpi was lower in F8-polysaccharide-pretreated than $\mathrm{H}_{2} \mathrm{O}$-pretreated plants in our three repeats. Inconsistent induction of $P R-4$ (JA marker gene) and Osmin (ET marker gene) was observed in the three repeats. Therefore, the expression of $P R-4$ and Osmin genes in $N$. benthamiana is not related to F8-polysaccharide treatment. The expression of $P R-1, P R-2, P D F-1.2$, and $E R E B P$ was consistent in our repeats (Fig. 9A to D). A comparison of $\mathrm{H}_{2} \mathrm{O}$ and F8-polysaccharide-pretreated plants over different times showed that the expression of $P R-1$ was highly induced at $12 \mathrm{hpt}$
A

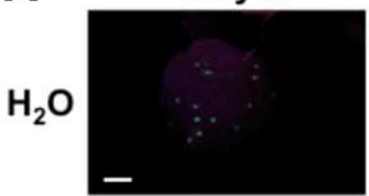

SA

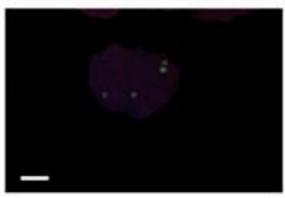

VB

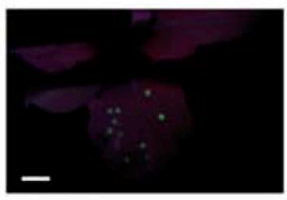

F8

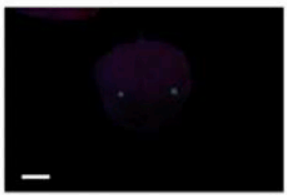

B

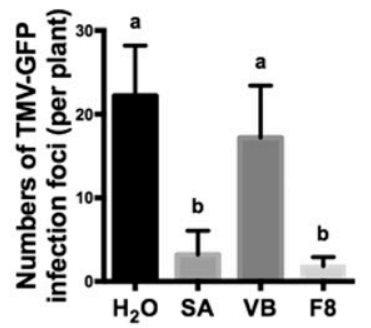

Day 7
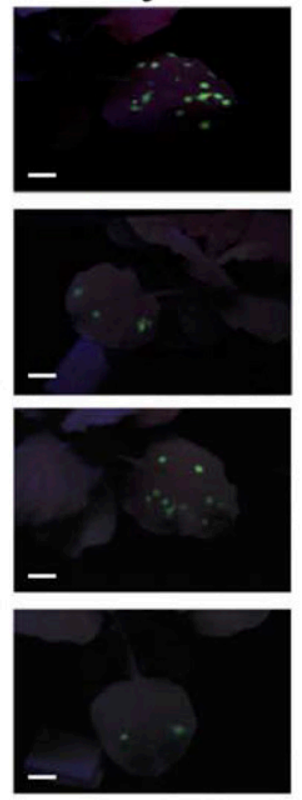

C

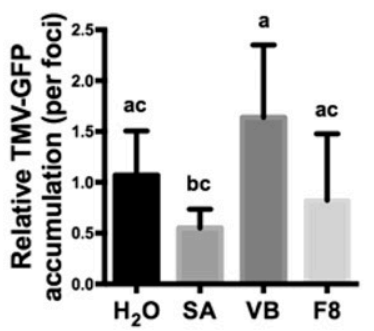

E

SA

VB

F8
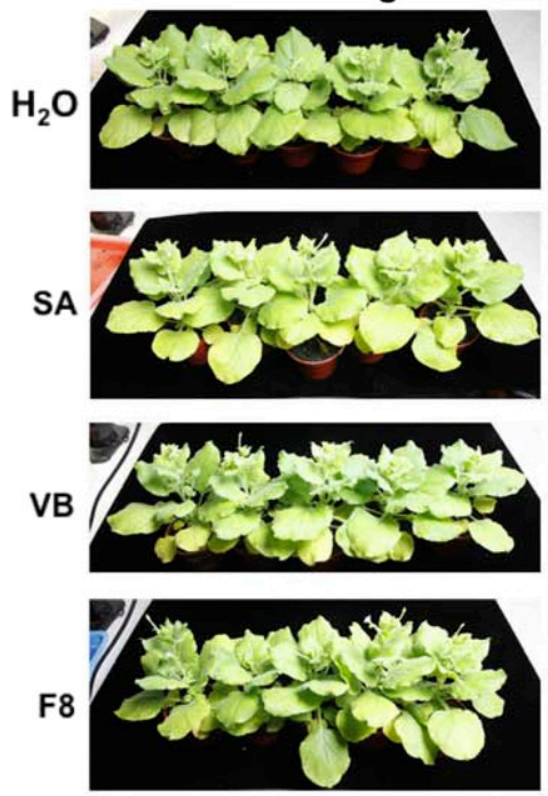

D

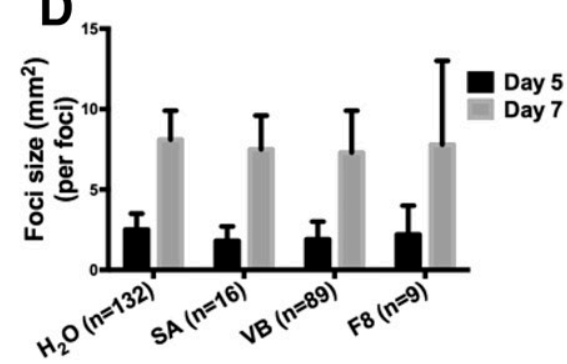

UV light
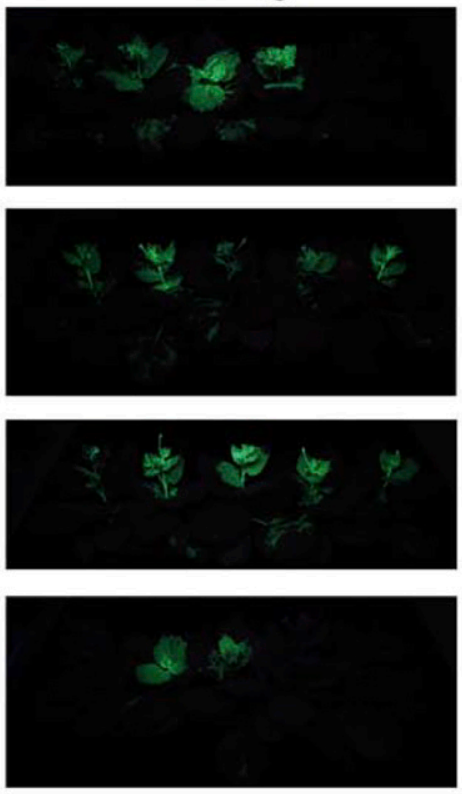

F

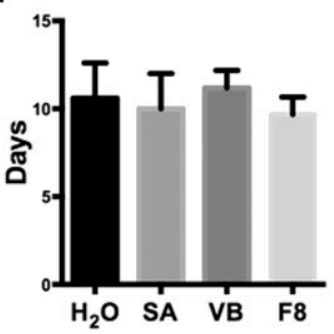

Fig. 5. Nicotiana benthamiana infected with Tobacco mosaic virus tagged with green fluorescence protein (TMV-GFP). A, N. benthamiana was pretreated with $\mathrm{H}_{2} \mathrm{O}, 5 \mathrm{mM}$ salicylic acid (SA), vegetable broth (VB), or F8 culture filtrate (F8) three times, with a 24-h interval between each spray. All plants were inoculated with TMV-GFP at $24 \mathrm{~h}$ posttreatment. Photos were taken under UV light at 5 and 7 days postinoculation (dpi). Scale bar $=2 \mathrm{~cm}$. B, The mean number of TMVGFP infection foci per plant at $5 \mathrm{dpi}$. Data are means \pm standard deviation (SD) from two to five plants. Different letters above the bars represent significant differences among groups, $P<0.05$ by one-way analysis of variance (ANOVA) with Tukey's posttest. C, The relative accumulation of TMV-GFP per foci. TMV-GFP was quantified by quantitative reverse transcription-polymerase chain reaction, and $\beta$-actin was an internal control. Data are means \pm SD from two to five plants. Different letters above the bars represent significant differences among groups, $P<0.05$ by one-way ANOVA with Tukey's posttest. D, The area (in square millimeters) of TMV-GFP infection foci was measured at 5 and 7 dpi. E, Photos of TMV-GFP-infected plants taken under white light or UV light at 13 dpi. F, The mean time (days) for TMV-GFP to move to apical leaves. Experiments were repeated at least three times with similar results. Data are means \pm SD from two to five plants. 
and gradually reduced (Fig. 9A). However, after TMV inoculation, the expression of $P R-1$ was highly induced in plants treated with F8-polysaccharide at 48 and 72 hpi (Fig. 9B). The expression pattern of $P R-2$ was similar to that of $P R-1$ with or without TMV inoculation (Fig. 9C and D). The expression of $P R-1$ and $P R-2$ show a typical priming expression pattern (Martinez-Medina et al. 2016).

$P D F-1.2$ was induced at $12 \mathrm{hpt}$ and $12 \mathrm{hpi}$ but with no significant difference between $\mathrm{H}_{2} \mathrm{O}$ - and F8-polysaccharidepretreated plants in our repeated experiments. No significant
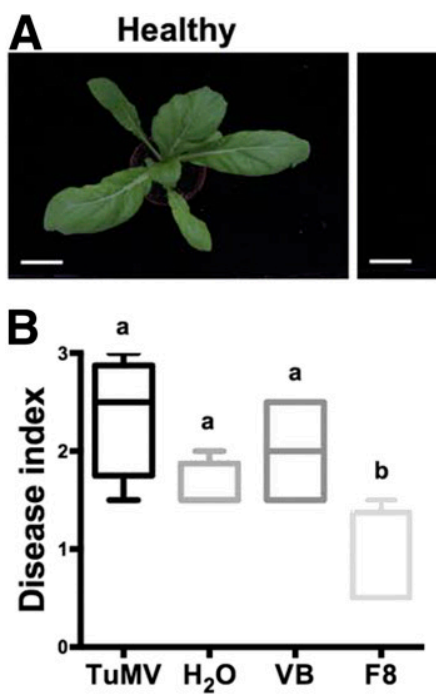

TuMV

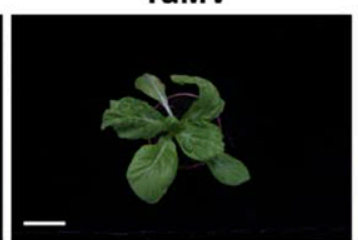

C

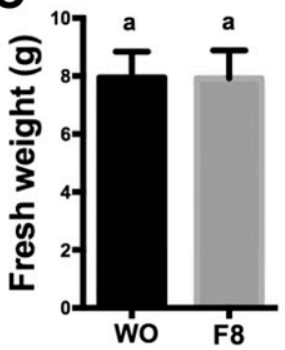

$\mathrm{H}_{2} \mathrm{O}$

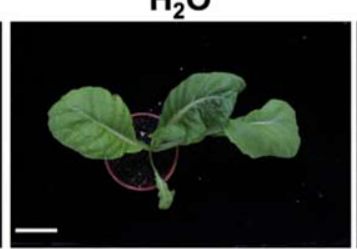

Nicotiana benthamiana

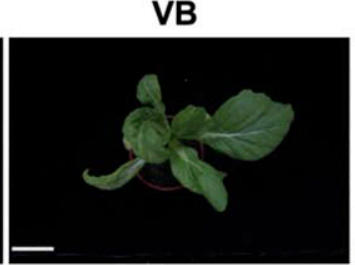

Brassica juncea
F8

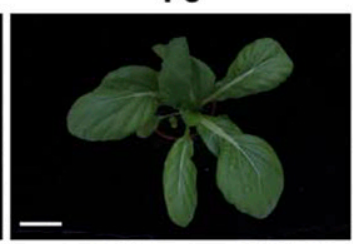

D
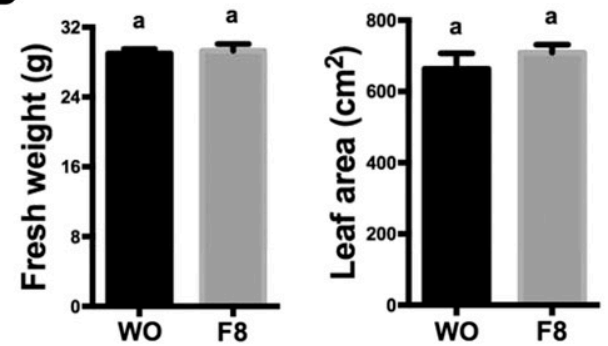

Fig. 6. Symptoms induced by Turnip mosaic virus (TuMV) in Brassica juncea pretreated with F8 culture filtrate and fitness cost analysis of Nicotiana benthamiana and Brassica juncea with F8 culture filtrate pretreatment. A, Brassica juncea was pretreated with $\mathrm{H}_{2} \mathrm{O}$, vegetable broth (VB), or F8 culture filtrate (F8) three times, with a $24-\mathrm{h}$ interval between each spray, then, were inoculated with TuMV at 24 h posttreatment. Photos were taken at 13 days postinoculation (dpi). Scale bar $=5 \mathrm{~cm}$. B, The disease index induced by TuMV was recorded at 13 dpi. Box plots represent medians, 25 to 75 percentiles (boxes), and minimum and maximum values (whiskers) from six plants. Different letters above the bars represent significant differences among groups, $P<0.05$ by one-way analysis of variance, with Tukey's posttest. Experiments were repeated three times with similar results. C, Shoots of six $N$. benthamiana (15 days after seeding) or D, Brassica juncea (10 days after seeding) without treatment (WO) or with F8 pretreatment were used to measure fresh weight and leaf area at 20 or 25 days posttreatment, respectively. All leaves were photographed with identical illumination and exposure conditions. The average leaf area per plant was calculated.

A

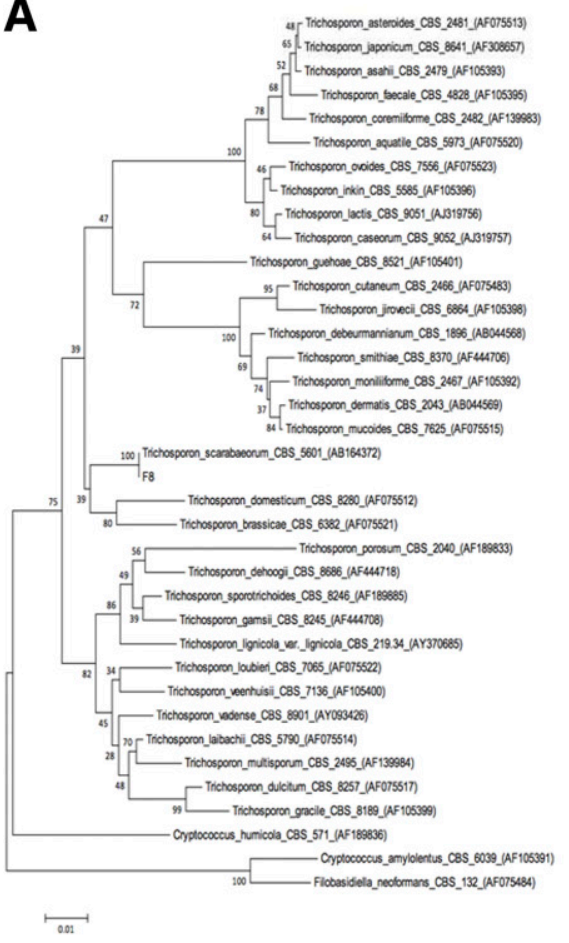

B

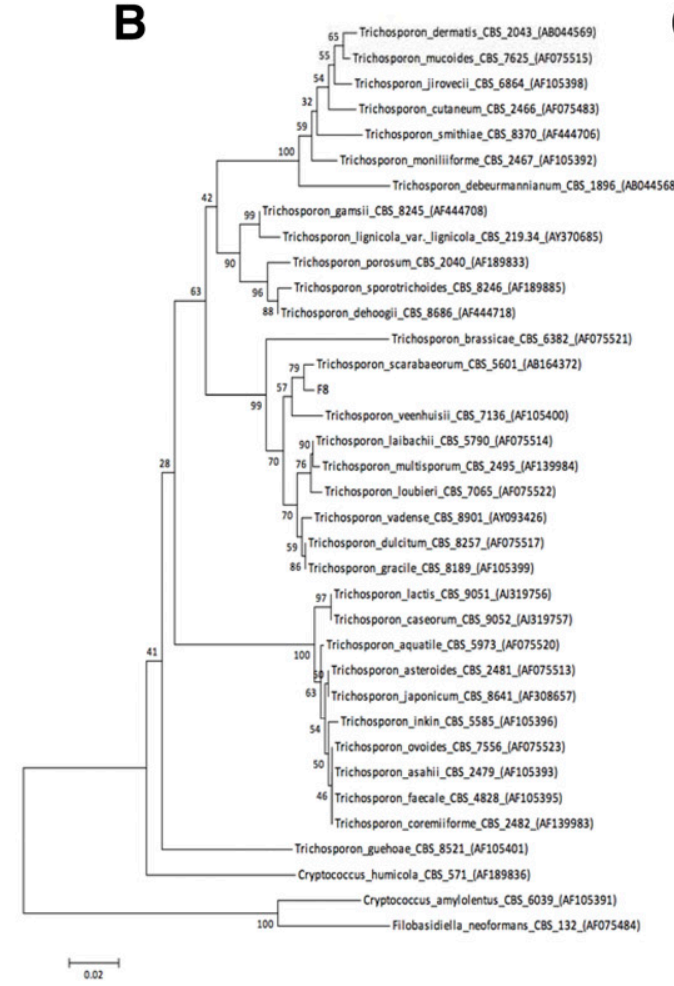

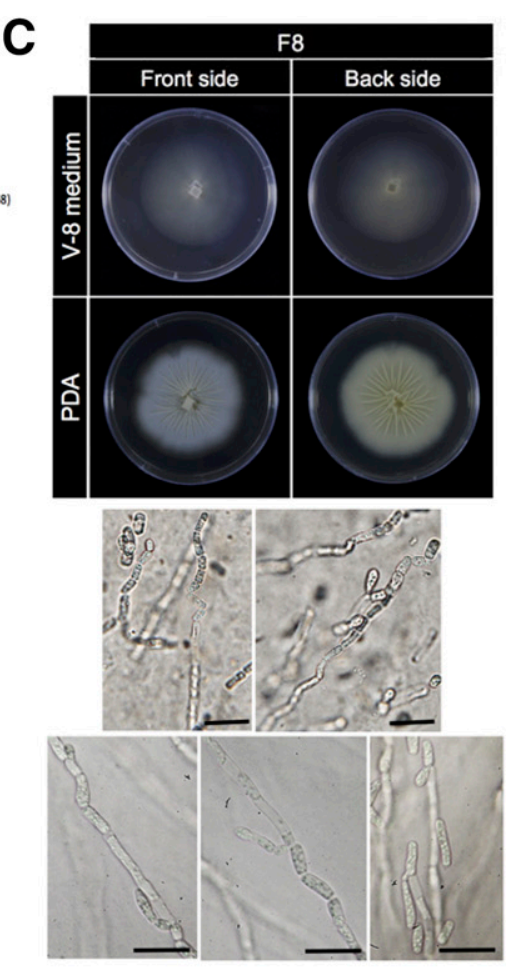

Fig. 7. Identification of F8 fungi. A, Phylogenetic tree of F8 fungi based on the D1/D2 region and B, intergenic transcribed spacer region by neighbor-joining and maximum likelihood methods. The results of both analysis methods was similar, and only the neighbor-joining tree is shown here. $\mathbf{C}$, F8 fungi were cultured on $10 \% \mathrm{~V}-8$ agar (V-8) and potato dextrose agar (PDA) plates for 15 days at $25^{\circ} \mathrm{C}$. Colony morphology was photographed and presented with front and back side. Microscopy depicting the morphology of arthroconidia and hyphae of $\mathrm{F} 8$ fungi grown on V-8 plate, $25^{\circ} \mathrm{C}$, for 7 days. Scale bar $=20 \mu \mathrm{m}$. 
induction of EREBP expression was observed in $\mathrm{H}_{2} \mathrm{O}$ - and $\mathrm{F} 8$ polysaccharide-pretreated plants in our repeats.

\section{DISCUSSION}

Our results successfully demonstrate that a natural product can induce prominent plant immunity in highly susceptible hosts, to confer effective resistance to virus infection without cost to the fitness of the plant. We successfully identified a culture filtrate derived from a fungi (F8) belonging to the genus Trichosporon within class Basidiomycete that greatly enhanced virus resistance in different susceptible plants against different viruses without obvious resistance-associated fitness cost. The F8 culture filtrate induced an unusual type of defense priming that differs from but is more effective than SA-induced resistance. On further fractionating the F8 culture filtrate, we identified a fraction of polysaccharide (F8-polysaccharide) composed of D-mannose, D-galactose, and D-glucose, in the ratio 1.0:1.2:10.0, with a $\alpha$-D-1,4-glucan linkage and an estimated molecular weight of 4.2 to $12.0 \mathrm{kDa}$, that is responsible for inducing plant resistance against viruses.

Several polysaccharides, including carrageenans ( $\alpha-1,3$ and $\beta$-1, 4-glycosidic), fucans ( $\alpha-1,4$ and $\alpha-1,3$ glycosidic), and laminarin ( $\beta-1,6-1,3$ glucans) have been reported to induce plant resistance against TMV in other local lesion hosts, including Nicotiana tabacum cv. Xanthi and N. tabacum L cv. Samsun H (Ghannam et al. 2013; Klarzynski et al. 2000, 2003;
Nagorskaia et al. 2008; Reunov et al. 2004; Vera et al. 2012). However, our data indicate that even though F2 and F8 culture filtrates induced similar resistance in $N$. glutinosa (Fig. 1A and B), only F8 culture filtrate induced prominent resistance in N. benthamiana (Fig. 1C and D). Therefore, substances that trigger resistance in local lesion hosts may not be able to trigger resistance in susceptible hosts and using one or more local lesion hosts alone for resistance analysis may not be sufficient for screening antiviral substances in susceptible plants.

An interesting phenomenon was that F8 culture filtrate did not induce typical SAR in $N$. benthamiana. F8 culture filtrate induced only local resistance and did not induce SAR. However, even though SA can induce SAR, SAR did not provide as effective protection against TMV as did F8 culture filtrate (Fig. 4). Although SAR can enhance plant resistance against a broad spectrum of pathogens, SAR induction may not be the best strategy for protecting plants against viruses.

Our tracking assay using TMV-GFP to inoculate $N$. benthamiana indicates that F8 culture filtrate mainly reduced the number of initial infection foci. Once TMV establishes the initial infection, TMV accumulation (per infection foci), and cell-to-cell and long-distance movement did not differ much between plants treated with $\mathrm{H}_{2} \mathrm{O}$ or $\mathrm{F} 8$ culture filtrate. Our results are similar to previous reports showing that certain tobacco plants treated with mycolaminaran (a branched $\beta$-1,3-glucan purified from the cytoplasm of Phytophthora megasperma) also inhibited initial viral infection (Kopp et al. 1989; Zinnen et al. 1991).

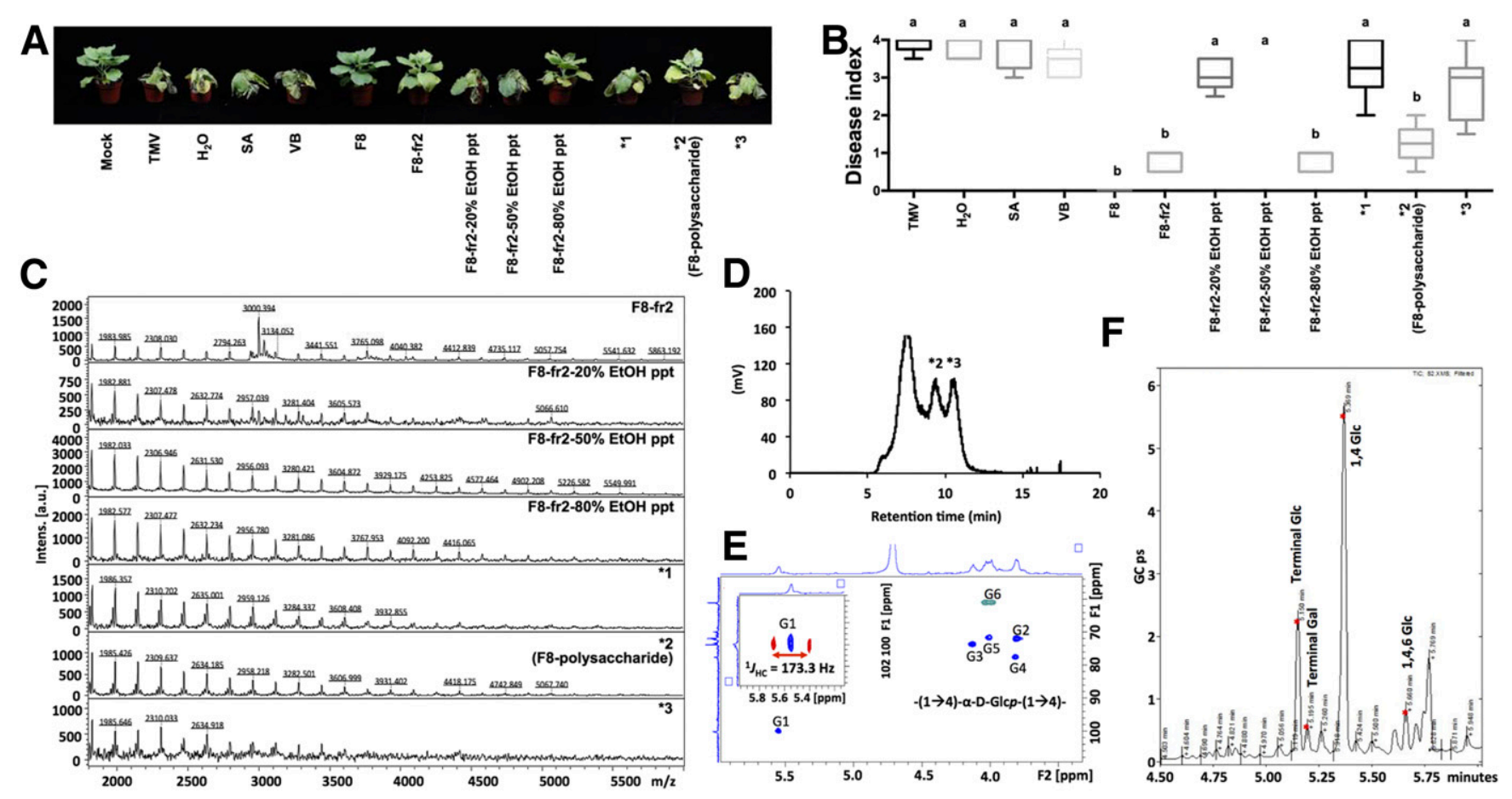

Fig. 8. Fractionation of F8 culture for antiviral activity analysis and identification, nuclear magnetic resonance, and linkage analysis of active functional compounds derived from F8 culture filtrate. Nicotiana benthamiana was pretreated with $\mathrm{H}_{2} \mathrm{O}, 5 \mathrm{mM}$ salicylic acid (SA), vegetable broth (VB), F8 culture filtrate (F8), F8-sephadex fraction 2 (F8-fr2), $\mathrm{H}_{2} \mathrm{O}$-resuspended pellets derived from F8-fr2 precipitated with 20, 50, and 80\% ethanol (F8-fr2-20\%, fr2-50, and fr2-80\% EtOH ppt), and high-performance liquid chromatography (HPLC)-purified peaks 1 to 3 (* 1 to $* 3$ ) from $\mathrm{H}_{2} \mathrm{O}-$ resuspended $\mathrm{F} 8$-fr2-80\% EtOH ppt three times, with a 24-h interval between each spray. We define peak *2 as F8-polysaccharide. Pretreated plants were inoculated with Tobacco mosaic virus (TMV) at $24 \mathrm{~h}$ posttreatment. A, Photos of TMV-inoculated plants taken at 7 days postinoculation (dpi). B, The disease index induced by TMV was recorded at 7 dpi. Box plots represent medians, 25 to 75 percentiles (boxes), and minimum and maximum values (whiskers) from six plants. Different letters above the bars represent significant differences among groups, $P<0.05$ by one-way analysis of variance, with Tukey's posttest. Experiments were repeated three times with similar results. C, Matrix-assisted laser desorption-ionization time of flight mass spectra of F8-sephadex fraction 2 (F8-fr2), $\mathrm{H}_{2} \mathrm{O}$-resuspended pellets derived F8-fr2$20 \%$ EtOH ppt, $50 \%$ EtOH ppt, and $80 \%$ EtOH ppt, and HPLC-purified peaks $* 1$ to $* 3$ from $\mathrm{H}_{2} \mathrm{O}-\mathrm{resuspended} \mathrm{F} 8$-fr2-80\% EtOH ppt at $m / z$ 1,800 to 6,000, where the most peaks are. D, HPLC-evaporative light scattering detection profile of F8-fr2-80\% EtOH ppt. Three major peaks, *1 to *3, were collected for matrix-assisted laser desorption-ionization time of flight mass spectra analysis shown in C. *2 was defined to be F8-polysaccharide. E, Heteronuclear single quantum coherence spectrum $\left({ }^{1} \mathrm{H}^{-13} \mathrm{C}\right.$ HSQC, $\left.\mathrm{D}_{2} \mathrm{O}, 323 \mathrm{~K}, 600 \mathrm{MHz}\right)$ of F8-polysaccharide. Determination of the ${ }^{1} J_{\mathrm{HC}}$ of $\mathrm{G} 1$ (alpha configuration) was based on the satellite signals of $\mathrm{G} 1\left(\mathrm{~d}_{\mathrm{H}} 5.63, \mathrm{~d}_{\mathrm{C}} 99.9\right)$ in the heteronuclear multiple bond correlation spectrum $\left({ }^{1} \mathrm{H}-{ }^{13} \mathrm{C} \mathrm{HMBC}\right)$. F, Gas chromatography mass spectrometry (GC-MS) linkage analysis of F8-polysaccharide. 1,4-linked-D-glucopyranosyl residue was the main linkage composition. 
Although we cannot rule out the possibility that F2 or F8 culture filtrate may inhibit virus infection, our data suggested that SA-governed immune responses are highly induced on F8 culture filtrate or F8-polysaccharide treated plants during TMV infection (Figs. 4F and 9B and D). The phenomenon we observed of low infection foci but similar virus accumulation per foci and similar cell-to cell and long distance movement between plants treated with $\mathrm{H}_{2} \mathrm{O}$ and $\mathrm{F} 8$ culture filtrate fits the proposed scheme for microbe-plant interactions during infection, in which microbe-encoded PAMPs can trigger the plant PTI to protect against pathogen infection and pathogens may encode one or more effectors to suppress PTI for successful infection (Jones and Dangl 2006). Recent reports indicated that plant viral double-stranded RNAs can act as PAMPs that trigger PTI responses against viral infection in Arabidopsis (Niehl et al. 2016). In addition, TMV replicase has been found to interact with and degrade a NAC domain transcription factor, ATAF2. Because ATAF2 can induce host defense responses, the accumulation of TMV replicase will cause gradual degradation of ATAF2, thus leading to an attenuated defense response during TMV infection (Wang et al. 2009). Our data suggest that F8 culture filtrate treatment greatly enhanced PTI.

Our analysis revealed that F8-polysaccharide is the major component in F8 culture filtrate for inducing plant resistance. F8polysaccharide induces a typical priming expression pattern of SAresponsive genes (Fig. 9A to D). A previous report demonstrated that priming involves fewer fitness costs (Conrath et al. 2015; Mauch-Mani et al. 2017; van Hulten et al. 2006; Wang et al. 2015), which may explain, in part, why F8 culture filtrate treatment did not confer any observable growth defect (Fig. 6C and D).

The origin of F8-polysaccharide is intriguing. The composition and structure have not been previously revealed. The F8polysaccharide is obviously not directly derived from original VB culture medium because VB did not induce effective resistance in plants as compared with F8 culture filtrate treatment (Figs. 1, 2, 3, and 6B). Also, F8 pellets (contain mostly F8 fungi cell debris) do not induce as effective a resistance as F8 culture filtrate on $N$. benthamiana. F8-polysaccharide may come from the fermentation process between VB and F8 fungi, but its origin remains elusive. Although F8-polysaccharide is the major compound identified from F8 culture filtrate, plant resistance induced by F8-polysaccharide is not as effective as F8 culture filtrate (Fig. 8; Supplementary Table S3). This suggests that substances other than F8-polysaccharide in the F8 culture filtrate may also play roles in enhancing plant resistance.

Our analysis revealed that F8-polysaccharide is the major component in F8 culture filtrate for inducing plant resistance. F8-polysaccharide induces a typical priming expression pattern of SA-responsive genes (Fig. 9A to D). A previous report demonstrated that priming involves fewer fitness costs (Conrath et al. 2015; Mauch-Mani et al. 2017; van Hulten et al. 2006; Wang et al. 2015), which may explain, in part, why F8 culture
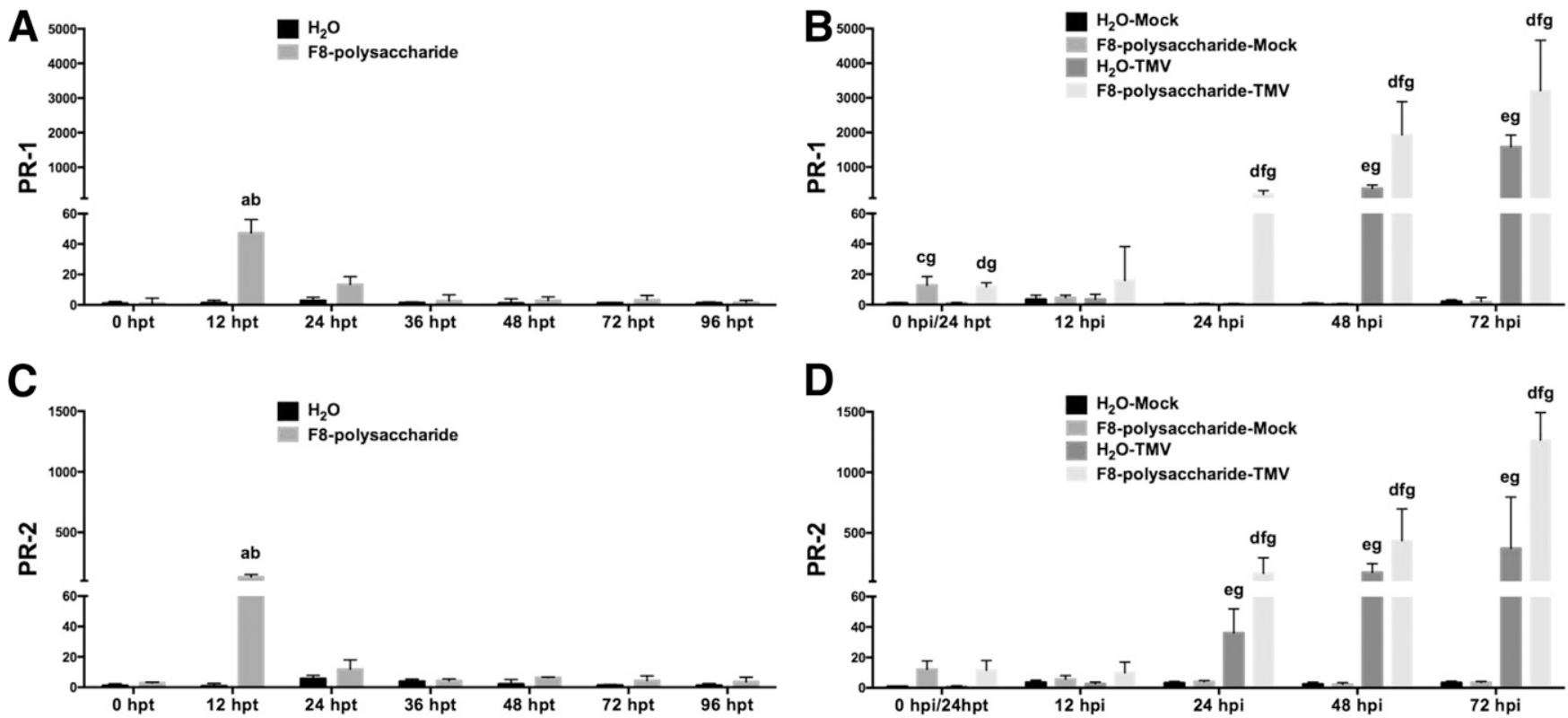

Fig. 9. The relative expression of salicylic acid (SA) associated immune marker genes. A, The relative expression of SA-responsive genes $P R-1$ and $\mathbf{C}, P R-2$ detected by quantitative reverse transcription-polymerase chain reaction (qRT-PCR). Plants were pretreated with $\mathrm{H}_{2} \mathrm{O}$ or $\mathrm{F} 8$-polysaccharide for total of three times, with a 24-h interval between each spray, then, samples collected at 0, 12, 24, 36, 48, 72, $96 \mathrm{~h}$ posttreatment (hpt). B and D, Tobacco mosaic virus (TMV) inoculation or mock treatment was conducted on different indicated 24-hpt plants. Leaf samples were collected from TMV-infected plants at $1,36,48$, and $72 \mathrm{~h}$ post-TMV inoculation. The relative expression of $P R-1$ (B) and $P R-2$ (D) was detected by qRT-PCR. Data are means \pm standard deviation from three to five plants. All lettering indicates statistical significance $(P<0.05$ by one-way analysis of variance, with Tukey's posttest). Letters indicate statistical significance as follows: a, between F8-polysaccharide-treated samples at indicated time to F8-polysaccharide-treated samples collected at 0 hpt; b, between $\mathrm{H}_{2} \mathrm{O}$ and F8-polysaccharide-treated samples at each time course; c, between $\mathrm{H}_{2} \mathrm{O}$-treated mock-inoculated and F8-treated mock-inoculated; d, between $\mathrm{H}_{2} \mathrm{O}$-treated TMV-inoculated and F8-treated TMV-inoculated; e, between $\mathrm{H}_{2} \mathrm{O}$-treated mock-inoculated and $\mathrm{H}_{2} \mathrm{O}$-treated TMV-inoculated; f, between F8-treated mockinoculated and F8-treated TMV-inoculated; g, indicates statistical significance to $\mathrm{H}_{2} \mathrm{O}$-treated mock-inoculated at $0 \mathrm{~h}$ postinoculation.

Table 3. ${ }^{1} \mathrm{H}$ and ${ }^{13} \mathrm{C}$ nuclear magnetic resonance chemical shifts $\left(\delta, \mathrm{D}_{2} \mathrm{O}, 323 \mathrm{~K}\right)$ of F8-polysaccharide

\begin{tabular}{|c|c|c|c|c|c|c|}
\hline$(1 \rightarrow 4)-\alpha-D-G I c p-(1 \rightarrow 4)-{ }^{a}$ & 1 & 2 & 3 & 4 & 5 & 6 \\
\hline${ }^{1} \mathrm{H}$ & 5.63 & 3.88 & 4.21 & 3.90 & 4.10 & $4.08 ; 4.11$ \\
\hline${ }^{13} \mathrm{C}$ & 99.9 & 71.8 & 73.6 & 77.4 & 71.8 & 60.8 \\
\hline
\end{tabular}

a The configuration was determined based on the ${ }^{1} J_{\mathrm{H} 1-\mathrm{C} 1}=173.3 \mathrm{~Hz}$. 
filtrate treatment did not confer any observable growth defect (Fig. 6C and D).

The origin of F8-polysaccharide is intriguing. The composition and structure have not been previously revealed. The F8-polysaccharide is obviously not directly derived from original VB culture medium because VB did not induce as effective a resistance in plants, as compared with F8 culture filtrate treatment (Figs. 1 and 6B). Also, F8 pellets (contain mostly F8 fungi cell debris) do not induce as effective a resistance as F8 culture filtrate on $N$. benthamiana. F8-polysaccharide may come from the fermentation process between VB and F8 fungi, but its origin remains elusive.

Our results provide several new insights into the application of induced plant resistance against viruses, which will be important to further develop management strategies to protect plants against virus infection.

\section{MATERIALS AND METHODS}

\section{Isolation of soil microorganisms and preparation of culture filtrate for antiviral activity assay.}

Soil samples (10 to $20 \mathrm{~g}$ ) were collected from a depth of 5 to $10 \mathrm{~cm}$ in Taipei, Taiwan. Soil micoorganisms were isolated with selective medium as reported by Ko et al. (2010), except we adjusted the $\mathrm{pH}$ of $\mathrm{VB}$ to 4.7 for the culture of $\mathrm{F} 8$ fungi and the VB was filtrated through a $25-\mu \mathrm{m}$ filter before storing at $-80^{\circ} \mathrm{C}$.

\section{Plant materials and inoculation of viruses.}

Plants were grown in a growth chamber with 10,000 lux fluorescent light and a 16-h light and 8-h dark cycle. N. glutinosa ( 6 to 7 weeks old, eight- to ten-leaf stage), N. benthamiana ( 3 to 4 weeks old, five- to six-leaf stage) and Brassica juncea (14 days old, two to three true-leaf stage) were used for one or both treatment and inoculation. For virus inoculation, plants were dusted with 600-grit silicon carbide powder (SigmaAldrich, St. Louis) and were mechanically inoculated. Virus inoculum was prepared with $100 \mathrm{ml}$ of $0.1 \mathrm{M}$ potassium phosphate (KP) buffer $\left(61.5 \% 0.1 \mathrm{M} \mathrm{K}_{2} \mathrm{HPO}_{4}\right.$ and $38.5 \% 0.1 \mathrm{M}$ $\mathrm{KH}_{2} \mathrm{PO}_{4}, \mathrm{pH} 7.0$ ) mixed with $0.1 \mathrm{~g}$ of lyophilized leaves of plants infected with TMV (Figs. 1, 4A to E, 8, and 9; Supplementary Fig. S6) or TMV tagged with green fluorescence protein (TMV-GFP) (Fig. 5; Table 1) (Rabindran and Dawson 2001). Different concentrations of TMV-purified particle inoculum was diluted with $0.1 \mathrm{M} \mathrm{KP}$ buffer $(61.5 \% 0.1 \mathrm{M}$ $\mathrm{K}_{2} \mathrm{HPO}_{4}$ and $38.5 \% 0.1 \mathrm{M} \mathrm{KH}_{2} \mathrm{PO}_{4}$, pH 7.0) (Figs. 2, 3, and 4F; Table 2). TuMV inoculum was prepared with $0.1 \mathrm{~g}$ of fresh leaves from plants infected with TuMV ground with $10 \mathrm{ml}$ of $0.1 \mathrm{M}$ sodium phosphate buffer $\left(0.1 \mathrm{M} \mathrm{NaH}_{2} \mathrm{PO}_{4}, 0.1 \mathrm{M}\right.$ $\mathrm{Na}_{2} \mathrm{HPO}_{4}$, and $0.5 \% \mathrm{Na}_{2} \mathrm{SO}_{3}, \mathrm{pH} 7.4$ ). The severity of TMV or TuMV symptoms of infected plants were scored based on external phenotype (Supplementary Fig S6). Transmission electron microscope (Philips Electronic Instruments, Mahwah, NJ, U.S.A.) was used to examine TMV particles of different inoculums.

\section{Purification of TMV particles.}

Leaves of TMV-infected $N$. benthamiana were harvested approximately 5 days after inoculation. The initial purification of TMV virus particles followed the protocol described by Gooding and Hebert (1967), with modifications. Briefly, polyethylene glycol with molecular weight 8,000 (PEG 8000) (SigmaAldrich) was used instead of PEG 6000. In addition, the semipurified preparation was submitted to linear density sucrose gradient (0 to $18 \%$ ) and was ultracentrifuged at $228,000 \times g$ for $1.5 \mathrm{~h}$. The light scattering region was collected and diluted with $100 \mathrm{ml}$ of suspension buffer $\left(35 \mathrm{mM} \mathrm{Na} \mathrm{HPO}_{4}, 15 \mathrm{mM}\right.$ $\mathrm{NaH}_{2} \mathrm{PO}_{4}$, and $0.05 \%$ 2-mercaptoethanol, $\left.\mathrm{pH} 7.2\right) . \mathrm{NaCl}(0.4 \mathrm{~g})$ and PEG 8000 (4 g) were added and were stirred for at least $1 \mathrm{~h}$ on ice, then, centrifuged at $9,000 \times g$ for $15 \mathrm{~min}$ at $4^{\circ} \mathrm{C}$. The pellets were resuspended in $1 \mathrm{ml}$ of suspension buffer. The concentration of the purified TMV suspension was determined $\left(A_{260}=0.3\right.$ for $1 \mathrm{mg}$ of TMV per milliliter) with a Nanodrop (ND-1000; Thermo Fisher Scientific, Waltham, MA, U.S.A.).

\section{Antiviral activity assays.}

Whole plants were sprayed with $0.4 \mathrm{ml}$ of $\mathrm{H}_{2} \mathrm{O}$, VB, culture filtrate derived from microorganisms, or $5 \mathrm{mM}$ SA (SigmaAldrich) three times, with a 24-h interval between each spray. Twenty-four hours after the final treatment, plants were rubbed with the virus inoculum.

\section{Measurement of area of TMV-GFP infection foci.}

Photographs of TMV-GFP infection foci in N. benthamiana leaves were taken under UV light at 5 and $7 \mathrm{dpi}$, and the level of fluorescence as well as the areas of fluorescent infection sites in each leaf were measured, using ImageJ v1.47. All imaging was conducted under identical illumination and exposure conditions to allow for comparisons. Mean values of areas of all infection foci per treatment were calculated.

\section{RNA extraction and gene expression analysis.}

Total RNA was isolated by use of Trizol (Invitrogen, Bethesda, MD, U.S.A.), according to the manufacturer's instruction. Residual DNA was removed using the Turbo DNA-free kit (Ambion, Austin TX, U.S.A.). cDNA was synthesized using the PrimeScript RT-PCR kit (Takara Bio, Shiga, Japan). qRT-PCR involved the SYBR protocol (Life Technologies, Gaithersburg, MD, U.S.A.) and the ABI 7100 real-time PCR system (Applied Biosystems, Carlsbad, CA, U.S.A.). For quantitative analysis, actin was used as an input control. Relative fold change in mRNA expression was determined by calculating cycle threshold $\left(2^{-\Delta \Delta C t}\right)$.

\section{Identification of fungi and phylogenetic analysis.}

Genomic DNA was extracted by the CTAB method (Chang et al. 1993). Internal transcribed spacer or 26S rDNA region sequences were amplified with specific primers designed for the ITS1-5.8S-ITS2 region (ITS5e-F and ITS4e-R) and the D1/D2 domain (LR1-F and LR3-R), respectively. The sequence alignment involved use of CLUSTAL X (1.81) (Thompson et al. 1997). All the phylogenetic trees in this study were generated using MEGA 5 (Tamura et al. 2011). Phylogenetic analyses involved $\mathrm{NJ}$ and ML methods. The branch supports of NJ and ML methods were analyzed by using PAUP* $4.0 \mathrm{v} 10$ (Swofford 2003) with bootstrapping. NJ involved a Kimura 2-parameter model and node support was analyzed by bootstrapping with 1,000 replicates. ML involved a Jukes-Cantor model and bootstrapping with 1,000 replicates. Bootstrap minimal concordance was set to $80 \%$. These sequence data have been submitted to the GenBank databases under accession number MG757354 and MG752969.

\section{Measurement of leaf area and fresh weight.}

$N$. benthamiana (16 days after seeding) and Brassica juncea (10 days after seeding) were treated with the F8 culture filtrate, as described in the antiviral activity assay. All shoot tissues of individual plants were measured for fresh weight. Leaves were randomly collected from six individual N. benthamiana or Brassica juncea plants with or without F8 culture filtrate treatment at 20 or 25 days posttreatment, respectively. Photos were taken from every collected leaf using identical illumination and exposure conditions. Each leaf area was measured using Image $\mathrm{J}$. 


\section{Identifying the active functional compound} of $F 8$ culture filtrate for inducing plant resistance.

To purify and characterize the active functional compound, freeze-dried powder (from $90 \mathrm{ml}$ of F8 culture filtrate) was dissolved in $90 \mathrm{ml}$ of distilled $\mathrm{H}_{2} \mathrm{O}$. After centrifugation at 6,000 × $g$ for $10 \mathrm{~min}$, the supernatant was separated on a prepacked gel filtration Sephadex LH-20 column (GE Healthcare Life Sciences, Uppsala, Sweden) eluted with $500 \mathrm{ml}$ of $\mathrm{H}_{2} \mathrm{O}$ per fraction. Eight fractions derived from the F8 culture filtrate (F8-fr1 to F8-fr8) were collected and freeze-dried, then, were dissolved in $90 \mathrm{ml}$ of distilled $\mathrm{H}_{2} \mathrm{O}$ and were tested for antiviral activity.

\section{Isolation of polysaccharides.}

The F8-fr was mixed with a ratio of ethanol to $\mathrm{H}_{2} \mathrm{O}$ /ethanol (80:20 [vol/vol]) and was stirred vigorously at $4^{\circ} \mathrm{C}$. The precipitate was centrifuged at $6,000 \times g$ for $10 \mathrm{~min}$. The supernatant was precipitated again by the addition of a ratio of ethanol to $\mathrm{H}_{2} \mathrm{O} / \mathrm{ethanol}(50: 50[\mathrm{vol} / \mathrm{vol}])$ and $\mathrm{H}_{2} \mathrm{O} /$ ethanol (20: $80[\mathrm{vol} / \mathrm{vol}])$. All the precipitate was dissolved in $90 \mathrm{ml}$ of distilled $\mathrm{H}_{2} \mathrm{O}$, then, tested for antiviral activity. To remove minor impurities, the $\mathrm{F} 8$-fr2-80\% EtOH pellets were further purified by preparative HPLC with a TSKgel P2500GW column $(21.5 \mathrm{~mm} \times 60 \mathrm{~cm}$; Tosoh Bioscience LLC, Tokyo) for structure elucidation. The HPLC-purified F8-frs were dissolved in distilled $\mathrm{H}_{2} \mathrm{O}$, then, tested for antiviral activity.

\section{Polysaccharide structure elucidation.}

For sugar composition analysis, the purified polysaccharide underwent methanolysis by $3 \mathrm{~N}$ Methanolic-HCl (Supelco, Bellefonte, PA, U.S.A.) for $14 \mathrm{~h}$ at $85^{\circ} \mathrm{C}$ in a sealed glass tube. After drying with $\mathrm{N}_{2}$, the residue was then acetylated with $50 \mu \mathrm{l}$ acetic anhydride and $10 \mu \mathrm{l}$ pyridine in $500 \mu \mathrm{l}$ methanol at room temperature for $20 \mathrm{~min}$. All solvent was removed by a steady stream of nitrogen, then, the Sylon HTP kit (Supelco) was used for trimthylation at room temperature for $30 \mathrm{~min}$. The dried sample was dissolved with $800 \mu \mathrm{l}$ of $n$-hexane (HPLC grade; Sigma) and, then, analyzed by GC-MS. All data were collected by GC-MS (Bruker) with a HP-5MS silica capillary column (30 m $\times 0.25 \mathrm{~mm}$ I.D., Agilent) and temperature gradient of $1 \mathrm{~min}$ at $60^{\circ} \mathrm{C}, 2 \mathrm{~min}$ at 60 to $90^{\circ} \mathrm{C}, 9 \mathrm{~min}$ at 90 to $290^{\circ} \mathrm{C}, 5 \mathrm{~min}$ at $290^{\circ} \mathrm{C}, 36 \mathrm{~min}$ at 290 to $300^{\circ} \mathrm{C}$ and kept for $2 \mathrm{~min}$ at $300^{\circ} \mathrm{C}$.

For the linkage analysis, the polysaccharide was lyophilized, then dissolved in $1 \mathrm{ml}$ of dimethyl sulfoxide (DMSO) overnight at $80^{\circ} \mathrm{C}$. $\mathrm{NaOH}$ pellets were ground with a motor and were added into the sample solution for a 2-h incubation in a sealed tube. The sample was methylated by using $\mathrm{CH}_{3} \mathrm{I}$ and powder $\mathrm{NaOH}$ in DMSO. Furthermore, the dried sample was partitioned with $\mathrm{H}_{2} \mathrm{O}$ and $\mathrm{CHCl}_{3}$ to give the organic layer. The dried organic layer residue was further hydrolyzed with $2 \mathrm{M}$ trifluoroacetic acid (TFA) at $120^{\circ} \mathrm{C}$ for $1 \mathrm{~h}$, to give the partially methylated monosaccharides. Then, the sample was reduced by $\mathrm{NaBD}_{4}$ at room temperature for $1 \mathrm{~h}$ and was neutralized with $\mathrm{AcOH}$. The solution was coevaporated with $\mathrm{MeOH}$ to remove the boric acid. After that, the sample was acetylated with $1: 2$ pyridine- $-\mathrm{Ac}_{2} \mathrm{O}$ at $80^{\circ} \mathrm{C}$ for $1 \mathrm{~h}$ and the mixture was partitioned with $\mathrm{H}_{2} \mathrm{O}-\mathrm{CHCl}_{3}$. The organic layer containing methyl alditol acetates was analyzed by GC-MS on a HP-5MS silica capillary column $(30 \mathrm{~m} \times 0.25 \mathrm{~mm}$ I.D., Agilent) temperature gradient of $1 \mathrm{~min}$ at $60^{\circ} \mathrm{C}, 2 \mathrm{~min}$ at 60 to $90^{\circ} \mathrm{C}, 9 \mathrm{~min}$ at 90 to $290^{\circ} \mathrm{C}, 5 \mathrm{~min}$ at $290^{\circ} \mathrm{C}, 36 \mathrm{~min}$ at 290 to $300^{\circ} \mathrm{C}$ and kept for $2 \mathrm{~min}$ at $300^{\circ} \mathrm{C}$. The polysaccharide consisted of terminal-D-glucopyranosyl residue, 4-linked-D-glucopyranosyl residue, and 4,6-linked-D-gluctopyranosyl residue.

The one- and two-dimensional (2D) NMR spectra were collected using a Bruker AVANCE 600 spectrometer in $\mathrm{D}_{2} \mathrm{O}$ at 323 K. All 2D NMR experiments were carried out with standard pulse sequences provided by Bruker. For the NMR data, the major composition of the polysaccharide was determined to be $\alpha-1,4$-glucan. The assignments of protons and carbons of the sample are given in Table 3.

\section{MALDI-TOF MS.}

A 1- $\mu$ l sample was spotted on a MTP 384 ground steel plate and $1 \mu \mathrm{l}$ of saturated universal matrix solution (Sigma) (dissolved in $50 \%$ acetonitrile with $0.1 \%$ TFA) was spotted on top of the sample for MALDI-TOF MS analysis. A Bruker Autoflex Speed MALDI-TOF/TOF MS (Bruker, Bremen, Germany) equipped with a Smartbeam laser $(1,000 \mathrm{~Hz})$ was used for MALDI-TOF MS analysis. All data were collected in positive ion liner mode and were analyzed using Daltonics flexAnalysis 3.0 (Bruker).

\section{ACKNOWLEDGMENTS}

We thank W.-H. Ko for help in establishing the soil microorganism isolation system.

\section{LITERATURE CITED}

Alexandersson, E., Mulugeta, T., Lankinen, Å., Liljeroth, E., and Andreasson, E. 2016. Plant resistance inducers against pathogens in Solanaceae species-From molecular mechanisms to field application. Int. J. Mol. Sci. 17:1673.

Bektas, Y., and Eulgem, T. 2015. Synthetic plant defense elicitors. Front. Plant Sci. 5:804.

Boller, T., and Felix, G. 2009. A renaissance of elicitors: Perception of microbe-associated molecular patterns and danger signals by patternrecognition receptors. Annu. Rev. Plant Biol. 60:379-406.

Chang, S., Puryear, J., and Cairney, J. 1993. A simple and efficient method for isolating RNA from pine trees. Plant Mol. Biol. Report. 11:113-116.

Chisholm, S. T., Coaker, G., Day, B., and Staskawicz, B. J. 2006. Hostmicrobe interactions: Shaping the evolution of the plant immune response. Cell 124:803-814

Conrath, U. 2011. Molecular aspects of defence priming. Trends Plant Sci. 16:524-531.

Conrath, U., Beckers, G. J., Langenbach, C. J., and Jaskiewicz, M. R. 2015. Priming for enhanced defense. Annu. Rev. Phytopathol. 53:97-119.

Cortes-Barco, A. M., Goodwin, P. H., and Hsiang, T. 2010. A comparison of induced resistance activated by benzothiadiazole, (2R,3R)-butanediol and an isoparaffin mixture against anthracnose of Nicotiana benthamiana. Plant Pathol. 59:643-653.

Dangl, J. L., and Jones, J. D. 2001. Plant pathogens and integrated defence responses to infection. Nature 411:826-833.

Dodds, P. N., and Rathjen, J. P. 2010. Plant immunity: Towards an integrated view of plant-pathogen interactions. Nat. Rev. Genet. 11:539-548.

Durrant, W. E., and Dong, X. 2004. Systemic acquired resistance. Annu. Rev. Phytopathol. 42:185-209.

Galvez, L.C., Banerjee, J., Pinar, H., and Mitra, A. 2014. Engineered plant virus resistance. Plant Sci. 228:11-25.

Ghannam, A., Abbas, A., Alek, H., Al-Waari, Z., and Al-Ktaifani, M. 2013. Enhancement of local plant immunity against tobacco mosaic virus infection after treatment with sulphated-carrageenan from red alga (Hypnea musciformis). Physiol. Mol. Plant Pathol. 84:19-27.

Glazebrook, J. 2005. Contrasting mechanisms of defense against biotrophic and necrotrophic pathogens. Annu. Rev. Phytopathol. 43:205-227.

Göhre, V., and Robatzek, S. 2008. Breaking the barriers: Microbial effector molecules subvert plant immunity. Annu. Rev. Phytopathol. 46:189-215

Gooding, G. V., Jr., and Hebert, T. T. 1967. A simple technique for purification of Tobacco mosaic virus in large quantities. Phytopathology 57:1285.

Gozzo, F., and Faoro, F. 2013. Systemic acquired resistance (50 years after discovery): Moving from the lab to the field. J. Agric. Food Chem. 61: 12473-12491.

Hammerschmidt, R. 1999. Induced disease resistance: How do induced plants stop pathogens? Physiol. Mol. Plant Pathol. 55:77-84.

Herring, R. J. 2008. Opposition to transgenic technologies: Ideology, interests and collective action frames. Nat. Rev. Genet. 9:458-463.

Jones, J. D., and Dangl, J. L. 2006. The plant immune system. Nature 444: 323-329.

Klarzynski, O., Descamps, V., Plesse, B., Yvin, J. C., Kloareg, B., and Fritig, B. 2003. Sulfated fucan oligosaccharides elicit defense responses in tobacco and local and systemic resistance against tobacco mosaic virus. Mol. Plant-Microbe Interact. 16:115-122. 
Klarzynski, O., Plesse, B., Joubert, J. M., Yvin, J. C., Kopp, M., Kloareg, B., and Fritig, B. 2000. Linear beta-1,3 glucans are elicitors of defense responses in tobacco. Plant Physiol. 124:1027-1038.

Ko, W. H., Tsou, Y. J., Lin, M. J., and Chern, L. L. 2010. Activity and characterization of secondary metabolites produced by a new microorganism for control of plant diseases. N. Biotechnol. 27:397-402.

Kopp, M., Rouster, J., Fritig, B., Darvill, A., and Albersheim, P. 1989. Hostpathogen interactions: XXXII. A fungal glucan preparation protects Nicotianae against infection by viruses. Plant Physiol. 90:208-216.

Loebenstein, G. 2009. Local lesions and induced resistance. Adv. Virus Res. 75:73-117.

Martinez-Medina, A., Flors, V., Heil, M., Mauch-Mani, B., Pieterse, C. M. J., Pozo, M. J., Ton, J., van Dam, N. M., and Conrath, U. 2016. Recognizing plant defense priming. Trends Plant Sci. 21:818-822.

Mauch-Mani, B., Baccelli, I., Luna, E., and Flors, V. 2017. Defense priming: An adaptive part of induced resistance. Annu. Rev. Plant Biol. 68:485-512.

Milling, A., Babujee, L., and Allen, C. 2011. Ralstonia solanacearum extracellular polysaccharide is a specific elicitor of defense responses in wilt-resistant tomato plants. PLoS One 6:e15853.

Mishina, T. E., and Zeier, J. 2007. Pathogen-associated molecular pattern recognition rather than development of tissue necrosis contributes to bacterial induction of systemic acquired resistance in Arabidopsis. Plant J. 50:500-513.

Nagorskaia, V. P., Reunov, A. V., Lapshina, L. A., Ermak, I. M., and Barabanova, A. O. 2008. [Influence of kappa/beta-carrageenan from red alga Tichocarpus crinitus on development of local infection induced by tobacco mosaic virus in Xanthi-nc tobacco leaves]. Izv. Akad. Nauk Ser. Biol. 35:360-364.

Newman, M. A., Sundelin, T., Nielsen, J. T., and Erbs, G. 2013. MAMP (microbe-associated molecular pattern) triggered immunity in plants. Front. Plant Sci. 4:139.

Niehl, A., Wyrsch, I., Boller, T., and Heinlein, M. 2016. Double-stranded RNAs induce a pattern-triggered immune signaling pathway in plants. New Phytol. 211:1008-1019.

Pieterse, C. M., Van der Does, D., Zamioudis, C., Leon-Reyes, A., and Van Wees, S. C. 2012. Hormonal modulation of plant immunity. Annu. Rev. Cell Dev. Biol. 28:489-521.

Pieterse, C. M., Zamioudis, C., Berendsen, R. L., Weller, D. M., Van Wees, S. C., and Bakker, P. A. 2014. Induced systemic resistance by beneficial microbes. Annu. Rev. Phytopathol. 52:347-375.

Rabindran, S., and Dawson, W. O. 2001. Assessment of recombinants that arise from the use of a TMV-based transient expression vector. Virology 284:182-189.

Reunov, A., Nagorskaya, V., Lapshina, L., Yermak, I., and Barabanova, A. 2004. Effect of $\kappa / \beta$-Carrageenan from red alga Tichocarpus crinitus (Tichocarpaceae) on infection of detached tobacco leaves with tobacco mosaic virus. J. Plant Dis. Prot. 111:165-172.
Robert-Seilaniantz, A., Grant, M., and Jones, J. D. 2011. Hormone crosstalk in plant disease and defense: More than just jasmonate-salicylate antagonism. Annu. Rev. Phytopathol. 49:317-343.

Sastry, K. S., and Zitter, T. 2014. Management of virus and viroid diseases of crops in the tropics. Pages 149-480 in: Plant Virus and Viroid Diseases in the Tropics, Springer, Dordrecht, The Netherlands.

Scorzetti, G., Fell, J. W., Fonseca, A., and Statzell-Tallman, A. 2002. Systematics of basidiomycetous yeasts: A comparison of large subunit D1/D2 and internal transcribed spacer rDNA regions. FEMS Yeast Res. 2:495-517.

Swofford, D. L. 2003. PAUP*. Phylogenetic analysis using parsimony (*and other methods). Version 4. Sinauer Associates, Sunderland, MA, U.S.A.

Tamura, K., Peterson, D., Peterson, N., Stecher, G., Nei, M., and Kumar, S. 2011. MEGA5: Molecular evolutionary genetics analysis using maximum likelihood, evolutionary distance, and maximum parsimony methods. Mol. Biol. Evol. 28:2731-2739.

Thompson, J. D., Gibson, T. J., Plewniak, F., Jeanmougin, F., and Higgins, D. G. 1997. The CLUSTAL_X windows interface: Flexible strategies for multiple sequence alignment aided by quality analysis tools. Nucleic Acids Res. 25:4876-4882.

van Hulten, M., Pelser, M., van Loon, L. C., Pieterse, C. M., and Ton, J. 2006. Costs and benefits of priming for defense in Arabidopsis. Proc. Natl. Acad. Sci. U.S.A. 103:5602-5607.

Vera, J., Castro, J., Contreras, R. A., González, A., and Moenne, A. 2012. Oligocarrageenans induce a long-term and broad-range protection against pathogens in tobacco plants (var. Xanthi). Physiol. Mol. Plant Pathol. 79:31-39.

Wang, K., Liao, Y., Kan, J., Han, L., and Zheng, Y. 2015. Response of direct or priming defense against Botrytis cinerea to methyl jasmonate treatment at different concentrations in grape berries. Int. J. Food Microbiol. 194:32-39.

Wang, X., Goregaoker, S. P., and Culver, J. N. 2009. Interaction of the Tobacco mosaic virus replicase protein with a NAC domain transcription factor is associated with the suppression of systemic host defenses. J. Virol. 83:9720-9730.

Wiesel, L., Newton, A. C., Elliott, I., Booty, D., Gilroy, E. M., Birch, P. R., and Hein, I. 2014. Molecular effects of resistance elicitors from biological origin and their potential for crop protection. Front. Plant Sci. 5:655.

Zheng, X. Y., Zhou, M., Yoo, H., Pruneda-Paz, J. L., Spivey, N. W., Kay, S. A., and Dong, X. 2015. Spatial and temporal regulation of biosynthesis of the plant immune signal salicylic acid. Proc. Natl. Acad. Sci. U.S.A. 112: 9166-9173.

Zhu, F., Xi, D. H., Yuan, S., Xu, F., Zhang, D. W., and Lin, H. H. 2014. Salicylic acid and jasmonic acid are essential for systemic resistance against Tobacco mosaic virus in Nicotiana benthamiana. Mol. PlantMicrobe Interact. 27:567-577.

Zinnen, T. M., Heinkel, C. M., Hudspeth, M., and Meganathan, R. 1991. The role of cytoplasmic mycolaminaran in inhibiting initial viral infection of certain Nicotiana species. Phytopathology 81:426-428. 